\title{
Targeted profiling of human extrachromosomal DNA by CRISPR-CATCH
}

3 King L. Hung ${ }^{1}$, Jens Luebeck,3,\#, Siavash R. Dehkordi,\#, Ceyda Coruh4, Julie A. Law ${ }^{4}$,

4 William J. Greenleaf ${ }^{1,5}$, Paul Mischel $^{6}$, Vineet Bafna ${ }^{3}$, Howard Y. Chang ${ }^{1,7,}$

5

$6{ }^{1}$ Center for Personal Dynamic Regulomes, Stanford University, Stanford, CA 94305,

7 USA.

$8{ }^{2}$ Bioinformatics and Systems Biology Graduate Program, University of California at San

9 Diego, La Jolla, CA, 92093, USA

$10{ }^{3}$ Department of Computer Science and Engineering, University of California at San

11 Diego, La Jolla, CA, 92093, USA

$12{ }^{4}$ Plant Molecular and Cellular Biology Laboratory, Salk Institute for Biological Studies,

13 La Jolla, CA, 92037, USA.

$14{ }^{5}$ Department of Genetics, Stanford University School of Medicine, Stanford, CA.

$15{ }^{6}$ Department of Pathology, Stanford University School of Medicine, Stanford, CA

16 94305, USA.

$17{ }^{7}$ Howard Hughes Medical Institute, Stanford University School of Medicine, Stanford,

18 CA 94305, USA.

19

20 \# These authors contributed equally

21 * Correspondence should be addressed to: howchang@stanford.edu 
Hung et al., (CHANG), p. 2

\section{ABSTRACT}

23 Extrachromosomal DNA (ecDNA) is a common mode of oncogene amplification

24 but is challenging to analyze. Here, we present a method for targeted purification of

25 megabase-sized ecDNA by combining in-vitro CRISPR-Cas 9 treatment and pulsed field

26 gel electrophoresis of agarose-entrapped genomic DNA (CRISPR-CATCH). We

27 demonstrate strong enrichment of ecDNA molecules containing EGFR, FGFR2 and MYC

28 from human cancer cells. Targeted purification of ecDNA versus chromosomal DNA

29 enabled phasing of genetic variants and provided definitive proof of an EGFRvIII mutation

30 on ecDNA and wild-type EGFR on chromosomal DNA in a glioblastoma neurosphere

31 model. CRISPR-CATCH followed by nanopore sequencing enabled single-molecule

32 ecDNA methylation profiling and revealed hypomethylation of the EGFR promoter on

33 ecDNA compared to the native chromosomal locus in the same cells. Finally, separation

34 of ecDNA species by size and sequencing allowed accurate reconstruction of megabase-

35 sized ecDNA structures with base-pair resolution. CRISPR-CATCH is a new addition to

36 the toolkit for studying focal amplifications in cancer and will accelerate studies aiming to

37 explore the genetic and epigenetic landscapes of ecDNA. 
Hung et al., (CHANG), p. 3

\section{INTRODUCTION}

Oncogene amplification is a key cancer driving mechanism and frequently occurs on circular extrachromosomal DNA (ecDNA). ecDNA oncogene amplifications are present in half of human cancer types and up to one third of tumor samples and are associated with poor patient outcomes ${ }^{1-3}$. Given the prevalence of ecDNA in cancer, there is an urgent need for better characterization of unique genetic and epigenetic features of ecDNA in order to understand how it may differ from chromosomal DNA and obtain clues about how it is formed and maintained in tumors. However, isolation and targeted profiling of megabase-sized, clonal ecDNAs is currently challenging due to their large sizes and sequence complexity, in contrast to small kilobase- and subkilobase-sized DNA circles known as extrachromosomal circular DNA elements (eccDNAs) observed also in non-cancer cells and apoptotic byproducts ${ }^{4,5}$.

There are currently three main approaches to analyzing sequences of ecDNAs in cancer cells: 1) DNA fluorescence in situ hybridization (FISH), 2) bulk whole genome sequencing (WGS), and 3) exonuclease digestion of linear DNA followed by DNA amplification. The first method, DNA FISH, involves arresting cells in metaphase followed by chromosome spreading and hybridization of a DNA probe on a microscope slide. It provides excellent separation of ecDNA and chromosomal DNA signals and has been used to confirm the presence of oncogenes and drug resistance genes on ecDNA. However, this method is low throughput (tens of cells) and provides limited, binary sequence information (a probe either binds or does not bind to DNA). The second method, bulk short- or long-read sequencing, provides much higher sequence resolution. However, sequencing signal represents a combination of all DNA material in a sample, including ecDNA and chromosomal DNA. In addition to the ambiguous origin of sequencing reads, rearranged ecDNA sequences are computationally inferred ${ }^{1,6}$ but difficult to validate as sequencing reads are far too short to span the entire length of an ecDNA molecule (typically several megabases). Optical mapping (OM) allows analysis of longer DNA molecules (up to several hundred kilobases) by compromising nucleotidelevel information but each individual OM molecule is typically shorter than an ecDNA circle $^{7,8}$. Sequence segments can be computationally "stitched" together to form a list of candidate reconstructed paths, though empirically proving the true ecDNA structure, 
69 when possible, is very time-consuming and labor-intensive. The third method, 70 exonuclease treatment combined with DNA amplification, is effective for small DNA 71 circles (up to tens of kilobases; Circle-seq ${ }^{4,9}$ ) and was recently applied to ecDNA in cancer 72 cells $^{10}$. It entails magnetic bead-based DNA isolation, treatment with an exonuclease to 73 deplete linear DNA, followed by multiple displacement amplification (MDA). This method 74 requires intact DNA circles and is therefore highly limited by ecDNA size, as megabase75 sized DNA molecules are extremely fragile in solution and prone to breakage. Further, 76 this method requires DNA amplification and, therefore, cannot be used for epigenetic analyses. Phi29, the processive MDA polymerase, produces amplicons which are tens of kilobases and thus amplifies small circles via rolling-circle amplification; however, this is currently challenging for megabase-sized ecDNA. Finally, analysis of these enriched ecDNAs by short- or long-read sequencing also suffers from the same read length limitations for amplicon reconstruction.

Here we report a method for purifying megabase-sized ecDNA from cancer cells. DNA amplification is not required; thus, this method allows targeted analyses of both the genetic sequence and epigenetic features of purified ecDNA. We also provide an analytical pipeline for reconstructing amplicon structures de novo with high confidence using sequence information of ecDNA species separated by size.

\section{RESULTS}

\section{Purification and visualization of ecDNA by CRISPR-CATCH}

To preserve large intact circular ecDNA, we encapsulated genomic DNA of GBM39

91 cells (patient-derived glioblastoma neurosphere model containing EGFR ecDNA) in 92 agarose plugs (Methods). Fragment size distribution analysis by pulsed field gel 93 electrophoresis (PFGE) showed that virtually all agarose-entrapped genomic DNA 94 containing ecDNA was restricted to either the loading well or the upper compression zone 95 (CZ, region of large DNA molecules; Extended Data Figure 1a). ecDNAs are not 96 detectable in the resolution window, suggesting that intact circular ecDNAs do not migrate 97 freely in PFGE as suggested by previous southern blot studies ${ }^{11-13}$. To selectively pull 98 ecDNA into the resolution window of the gel, we pre-incubated GBM39 genomic DNA in 
100 amplified sequence on ecDNA. We reasoned that a single cut would linearize ecDNA, 101 resulting in differential migration in PFGE (Figure 1a). We further reasoned that the same 102 single cut in the corresponding chromosomal locus would result in two much larger 103 chromosomal DNA pieces that migrate much more slowly than ecDNA and therefore 104 would not be co-enriched. Cas9 digestion of EGFR ecDNA resulted in a prominent band 105 of 1.2-1.37 megabases (Figure 1b,c), concordant with the 1.258 Mb amplicon predicted 106 by AmpliconArchitect analysis of bulk WGS and AmpliconReconstructor analysis of OM 107 data ${ }^{7,8}$. Short-read sequencing of the gel-extracted band confirmed strong enrichment of 108 the expected ecDNA sequence (Figure 1d,e), demonstrating that a single cut is sufficient 109 to allow enrichment of ecDNA by PFGE. We refer to this method as CRISPR-CATCH 110 (Cas9-Assisted Targeting of CHromosome segments, a term previously coined for a two111 cut Cas9 treatment followed by gel extraction for isolating and cloning bacterial 112 chromosomal fragments ${ }^{14,15}$ ). CRISPR-CATCH enabled a 30 -fold enrichment of the 113 targeted ecDNA ( $60 \%$ of all sequencing reads vs. $2 \%$ in WGS), resulting in ultrahigh 114 ( 200x normalized) sequencing coverage (Figure 1d,e, ecDNA in Extended Data Figure 115 1b). Simultaneous cleavage of two sgRNA target sites $20 \mathrm{~kb}$ away from each other led to 116 loss of the sequence segment between the cut sites, as would be expected given a 117 circular structure and end-to-end junction of the amplified region (Figure 1f; ecDNA 118 guides $A+B)$. A single cut in the normal diploid chromosomal EGFR locus did not result 119 in a DNA band (as shown in Jurkat cells; Figure 1c), further supporting enrichment of 120 ecDNAs in GBM39 cancer cells. To isolate the chromosomal EGFR locus, we performed 121 CRISPR-CATCH using two sgRNAs targeting just outside of the amplified region 122 (upstream and downstream; Figure 1a,b). This dual-cut strategy resulted in a linear 123 fragment of roughly the same size as the ecDNA molecule and successfully enriched for 124 the chromosomal EGFR sequence as demonstrated by increased sequencing coverage 125 around the chromosome-targeting guides (Figure 1c,f; chromosomal DNA, Extended 126 Data Figure 1b). Chromosomal gel bands appeared much fainter than ecDNA bands 127 (Figure 1c), consistent with the fact that ecDNAs exist in higher copy numbers than the 128 chromosomal locus in GBM39 cells. Sequencing coverage analysis further validated 129 enrichment of ecDNA versus chromosomal DNA alleles (Extended Data Figure 1c,d). 130 Together, these results showed that CRISPR-CATCH can be used to isolate megabase- 
131 sized ecDNA molecules and corresponding chromosomal locus from the same cancer 132 cell sample. CRISPR-CATCH also provides the first empirical proof for amplicon size 133 predictions made by bulk sequencing analysis via migration patterns in PFGE.

\section{Phasing oncogenic mutations and single-nucleotide variants on ecDNA}

Next, we performed targeted analysis of the genetic sequences of ecDNA and chromosomal DNA containing the same oncogene locus (Figure 2a). Using ecDNA and 138 chromosomal DNA molecules containing the EGFR locus isolated using CRISPR$139 \mathrm{CATCH}$, we first identified structural variants using short-read sequencing data. GBM39 140 cells were previously shown to harbor the EGFRvlll deletion, an activating EGFR 141 mutation ${ }^{7,8,16}$. Importantly, sequencing coverage combined with breakpoint analysis of 142 CRISPR-CATCH data revealed that the EGFRvIII mutation is predominantly found on 143 ecDNA, while the chromosomal locus mainly contains full-length EGFR (Figure 2b). Full144 length EGFR appeared at $\sim 75 \%$ in the chromosomal fraction, consistent with the level of 145 chromosomal DNA enrichment (Figure 2b, Extended Data Figure 1d). This observation 146 suggests that the EGFRvIII mutation occurred after ecDNA formation and was strongly 147 selected subsequently (Figure 2c). This finding supports previous studies suggesting that 148 ecDNA may help cancer cells adapt to selective pressure and harbor unique genetic 149 alterations $\mathbf{s}^{6,17,18}$.

150 We then assessed the frequencies of single-nucleotide variants (SNVs) found on 151 purified ecDNA and chromosomal DNA. We identified SNVs most frequently observed on 152 ecDNAs, including clonal and subclonal variants (Figure 2d,e). Notably, we also found a 153 number of unique high-allele-frequency SNVs located in the chromosomal EGFR locus, 154 suggesting that the ecDNA and chromosomal loci may have diverged relatively early in 155 the mutational timeline (Figure 2d,e). Similar to the EGFRvIll analysis, unique SNVs 156 located in the chromosomal fraction exhibited allele frequencies of $70-75 \%$, consistent 157 with the level of chromosomal DNA enrichment (Figure 2d, Extended Data Figure 1d). 158 Low-frequency, subclonal ecDNA and chromosomal DNA mutations are indistinguishable 159 in bulk WGS data but can be clearly phased by CRISPR-CATCH (Figure 2e). 
We then examined the feasibility of analyzing epigenomic profiles of ecDNA using 163 CRISPR-CATCH. After ecDNA purification as before, we performed nanopore 164 sequencing to obtain single-molecule sequence information and DNA cytosine 165 methylation $(5 \mathrm{mC})$ profiles. We analyzed $5 \mathrm{mC}-\mathrm{CpG}$ methylation of purified ecDNA as a 166 proof of concept, and observed a strong anti-correlation of $5 \mathrm{mC}$ with chromatin 167 accessibility based on bulk ATAC-seq, validating the identification of regulatory elements 168 (Methods, Figure 3a,b, Extended Data Figure 2a). We also purified the corresponding 169 EGFR chromosomal locus in GBM39 cells and analyzed its DNA methylation profile 170 (Figure 3a,b). We observed reduced DNA methylation at regulatory elements on ecDNA 171 compared to the same elements on chromosomal DNA, suggesting altered gene 172 regulation (top 50 ATAC-seq peaks; Figure 3c). The four regions that lost $5 \mathrm{mC}$ on ecDNA 173 compared to its chromosomal locus in the same cells were all gene promoters, including 174 that of the EGFR oncogene (Methods, Figure 3d,e, Extended Data Figure 2b). The 175 pattern of hypomethylation corresponded to nucleosome positions shown by MNase-seq, 176 implying a more active chromatin state on ecDNA (Figure 3e, Extended Data Figure 177 2c) ${ }^{19,20}$. Finally, single-molecule analysis of purified ecDNA at the EGFR promoter 178 showed hypomethylation at the EGFR promoter and co-occurrence of methylation 179 spanning hundreds of CpG sites around the region on the same molecules (285 CpG 180 sites, Figure $\mathbf{3 f}$ ). Together, these data show that gene promoters on ecDNA may have 181 increased activities compared to the corresponding chromosomal locus on a single182 molecule level and demonstrate that CRISPR-CATCH can be used to measure 183 epigenomic features of ecDNA.

\section{De novo reconstruction of ecDNA amplicon structures}

Many cancer cells contain ecDNAs with more complex, heterogeneous structures,

187 including multiple sequence rearrangements and more than one circle species ${ }^{6}$. We 188 reasoned that CRISPR-CATCH may provide direct evidence of molecule size and 189 amplicon-phased structural information for these complex amplicons, and that this 190 information can be used to computationally reconstruct ecDNA with higher confidence.

191 To this end, we developed an analytical pipeline for de novo amplicon reconstruction from 192 CRISPR-CATCH data (Methods, Figure 4a). We modified and adopted 
193 AmpliconArchitect $^{6}$ for generating a copy-number-aware breakpoint graph for each

194 isolated amplicon. Next, we implemented a new method for extracting ecDNA candidate 195 paths from the graph, called Candidate AMplicon Path EnumeratoR (CAMPER). 196 Candidate ecDNA structures were generated from the breakpoint graph, estimated 197 multiplicity of genomic segments and molecular size based on PFGE using a depth-first 198 search (DFS) approach (Methods). Finally, quality estimates of resulting structures were 199 produced for filtering out any low-confidence reconstructions in the case of low-quality gel 200 extractions (e.g., incompletely separated ecDNA species) or undetectable breakpoints 201 from sequencing, etc. As validation, we reconstructed the 1.258 Mb circular ecDNA circle 202 encoding EGFR in GBM39 cells using this workflow, yielding a structure fully consistent 203 with previous reports using WGS and optical mapping ${ }^{7,8}$ (Extended Data Figure 3). To 204 further demonstrate the utility of this tool, we applied this pipeline to a stomach cancer 205 cell line, SNU16, which contains multiple ecDNA species with MYC, FGFR2 and 206 additional sequences connected by complex structural rearrangements (Extended Data 207 Figure 4a) ${ }^{21}$. CRISPR-CATCH using guides targeting the MYC or FGFR2 amplicon 208 resulted in multiple visible bands in PFGE (Figure 4b), revealing extensive molecular 209 heterogeneity of ecDNAs. Gel-extracted ecDNAs were multiplexed for sequencing. In 4 210 of 23 libraries, short-read sequencing of the CRISPR-CATCH-purified band was sufficient 211 to enable end-to-end, megabase-scale reconstruction of the ecDNA sequence. 5 libraries 212 corresponded to the compression zone and showed very low levels of ecDNA enrichment, 213 suggesting that the true ecDNA sizes are smaller than $2.2 \mathrm{Mb}$ (bands a,e,h,o,r, Figure 214 4b, Extended Data Figure 5). In the remaining cases, large amplicon sequences were 215 enriched, but one or more missing edges prevented unambiguous amplicon resolution 216 (Figure 4b, Extended Data Figure 5). From these data, we reconstructed three unique 217 ecDNAs containing MYC or FGFR2: a 1.604 Mb FGFR2 ecDNA that was reconstructed 218 from two independent CRISPR-CATCH treatments (using sgRNAs with cut sites $>300$ $219 \mathrm{~kb}$ apart), a smaller FGFR2 ecDNA species that was $228 \mathrm{~kb}$, and a $622 \mathrm{~kb}$ MYC ecDNA 220 containing sequences originating from chromosomes 8 and 11 (Figure 4c-e). All 221 reconstructions from CRISPR-CATCH data passing quality filters were supported by 222 contigs assembled from optical mapping data (N50 $50 \mathrm{Mb}$ ) provided to 223 AmpliconReconstructor ${ }^{7}$, further validating their structures (Methods, Figure 4c-e). 
224 Breakpoint graphs of ecDNA species were greatly simplified by CRISPR-CATCH 225 because each amplicon could be separately reconstructed and was not intermixed with 226 all other amplicons (Extended Data Figure 4b). Together, these data demonstrate the 227 utility of CRISPR-CATCH as a method for disambiguating ecDNA structure and size, 228 particularly when a heterogeneous mixture of ecDNAs is present. The method also aids 229 in accurate amplicon reconstruction orthogonal to contig assembly from bulk DNA.

\section{DISCUSSION}

By exploiting the distinctive PFGE migration pattern of large circular ecDNA, we

233 show that ecDNA can be purified from human cancer cells and separated by size using 234 CRISPR-CATCH. This method enables targeted analyses of ecDNA sequences and 235 epigenomic features that could not be previously achieved. CRISPR-CATCH also makes

236 it possible to directly compare ecDNA and the corresponding chromosomal locus in the 237 same cell sample by physically separating them. It is now possible to obtain allele-specific 238 information of ecDNA versus chromosomal DNA without relying on SNVs. Further, the 239 ability to phase SNVs by CRISPR-CATCH also enables identification of sequencing 240 signal originating from ecDNA in order to obtain allele-specific information (e.g. in bulk 241 RNA-seq data).

242 The scope and challenge of ecDNA isoforms were not fully appreciated in the past.

243 With the ability to separate ecDNA from the rest of the genome and accurately reconstruct 244 amplicon structures, CRISPR-CATCH may be applied to future studies on cancer cells 245 during early formation of ecDNA, cells evolving under chemotherapeutic or other selective 246 pressures, and in other settings where changes in genetic and chromatin features of 247 ecDNA are hypothesized to contribute to cancer cell evolution. As ecDNA often exhibits 248 tremendous structural heterogeneity, CRISPR-CATCH opens up a new window into 249 deciphering intratumoral genetic heterogeneity in cancer. The ability to separate ecDNAs 250 by size may provide increased structural resolution to other types of analysis, such as 251 single-cell sequencing, in which heterogeneous mixes of ecDNA structures are 252 computationally inferred but difficult to resolve confidently. These future applications of 253 CRISPR-CATCH may also address how ecDNA and chromosomal DNA diverge as they 254 evolve separately and under different kinetics. We note that tandem duplications on 
255 chromosomal DNA (e.g. homogeneously staining regions) can also be isolated by 256 CRISPR-CATCH with a single guide. Thus, CRISPR-CATCH should be used to 257 complement additional methods like metaphase FISH to verify the source of isolated 258 DNA.

259 We demonstrate that CpG methylation can be measured from purified ecDNA 260 molecules. Past studies have shown that cells containing ecDNA express amplified genes 261 at higher levels than cells containing linear amplifications, and that the ecDNA oncogene 262 locus is more accessible than other loci on linear DNA by bulk ATAC-seq ${ }^{1,8}$. Our 263 comparison of ecDNA versus chromosomal DNA encoding the same gene loci from the 264 same cells showed that gene promoters on circular ecDNA are less methylated than the 265 same promoters on linear chromosomal DNA, suggesting that ecDNA enables more 266 active transcription. In principle, CRISPR-CATCH may be coupled to several genomic 267 assays to understand key chromatin-templated processes on ecDNA such as 268 transcription, DNA replication, and repair ${ }^{22-24}$. CRISPR-CATCH presents an opportunity 269 for a multitude of molecular studies which will help elucidate how ecDNA oncogene 270 amplifications are regulated in cancer cells. 
Hung et al., (CHANG), p.11

\section{METHODS}

\section{Cell Culture}

274 GBM39 neurospheres were derived from patient tissue as previously described ${ }^{8}$. All other 275 cell lines used were obtained from ATCC. GBM39 cells were maintained in 276 DMEM/Nutrient Mixture F-12 (DMEM/F12 1:1; Gibco, Cat\# 11320-082), B-27 Supplement 277 (Gibco, Cat\# 17504044), 1\% penicillin-streptomycin (pen-strep; Thermo Fisher, Cat\# 278 15140-122), human epidermal growth factor (EGF, 20 ng/ml; Sigma-Aldrich, E9644), 279 human fibroblast growth factor (FGF, $20 \mathrm{ng} / \mathrm{ml}$; Peprotech) and Heparin (5 ug/ml; Sigma280 Aldrich, Cat\# H3149-500KU). SNU16 cells were maintained in DMEM/F12 supplemented 281 with $10 \%$ FBS and $1 \%$ pen-strep. All cells were cultured at $37^{\circ} \mathrm{C}$ with $5 \% \mathrm{CO}_{2}$. All cell 282 lines tested negative for mycoplasma contamination.

\section{Whole-Genome Sequencing}

285 Whole genome sequencing (WGS) data from bulk GBM39 cells were generated by a 286 previously published study ${ }^{8}$ and raw fastq reads obtained from the NCBI Sequence Read 287 Archive, under BioProject accession PRJNA506071. Reads were trimmed of adapter 288 content with Trimmomatic ${ }^{25}$ (version 0.39), aligned to the hg19 genome using BWA 289 MEM $^{26}$ (0.7.17-r1188), and PCR duplicates removed using Picard's MarkDuplicates 290 (version 2.25.3). WGS data from bulk SNU16 cells were previously generated 291 (SRR530826, Genome Research Foundation).

294 Genomic DNA was embedded in agarose plugs using a modified protocol based on 295 guidelines from the manufacturer of the CHEF Mapper XA System (Bio-Rad Laboratories) 296 as previously described ${ }^{27}$. Briefly, molten 1\% certified low melt agarose (Bio-Rad, 297 1613112) in PBS was equilibrated to $45^{\circ} \mathrm{C}$. 1 million cells were pelleted per condition, 298 washed twice with cold 1X PBS, resuspended in $30 \mathrm{ul} \mathrm{PBS}$, and briefly heated to $37^{\circ} \mathrm{C}$. $29930 \mathrm{ul}$ agarose solution was added to cells, mixed, transferred to a plug mold (Bio-Rad 300 Laboratories, Cat \#1703713) and incubated on ice for 10 minutes. Solid agarose plugs 301 containing cells were ejected into $1.5 \mathrm{ml}$ Eppendorf tubes, suspended in buffer SDE (1\% 302 SDS, $25 \mathrm{mM}$ EDTA at $\mathrm{pH}$ 8.0) and placed on shaker for 10 minutes. The buffer was 
303 removed and buffer ES (1\% N-laurolsarcosine sodium salt solution, $25 \mathrm{mM}$ EDTA at pH $3048.0,50 \mathrm{ug} / \mathrm{ml}$ proteinase K) was added. Agarose plugs were incubated in buffer ES at $30550^{\circ} \mathrm{C}$ overnight. On the following day, proteinase $\mathrm{K}$ was inactivated with $25 \mathrm{mM}$ EDTA 306 with $1 \mathrm{mM} \mathrm{PMSF}$ for 1 hour at room temperature with shaking. Plugs were then treated 307 with RNase A $(1 \mathrm{mg} / \mathrm{ml})$ in $25 \mathrm{mM}$ EDTA for 30 minutes at $37^{\circ} \mathrm{C}$, and washed with $25 \mathrm{mM}$ 308 EDTA with a 5-minute incubation. Plugs not directly used for ecDNA purification were 309 stored in $25 \mathrm{mM}$ EDTA at $4^{\circ} \mathrm{C}$.

To perform in-vitro Cas9 digestion, agarose plugs containing DNA were washed three 312 times with 1X NEBuffer 3.1 (New England BioLabs) with 5-minute incubations. Next, DNA 313 was digested in a reaction with $30 \mathrm{nM}$ single-guide RNA (sgRNA, Synthego) and $30 \mathrm{nM}$ 314 spCas9 (New England BioLabs, M0386S) after pre-incubation of the reaction mix at room 315 temperature for 10 minutes. To make two cuts on the native chromosomal locus, $15 \mathrm{nM}$ 316 of each sgRNA was added to the reaction. Cas9 digestion was performed at $37^{\circ} \mathrm{C}$ for 4 317 hours, followed by overnight digestion with $3 \mathrm{ul}$ proteinase $\mathrm{K}(20 \mathrm{mg} / \mathrm{ml})$ in a $200 \mathrm{ul}$ 318 reaction. On the following day, proteinase $\mathrm{K}$ was inactivated with $1 \mathrm{mM}$ PMSF for 1 hour 319 with shaking. plugs were then washed with 0.5X TAE buffer three times with 5-minute 320 incubations. Plugs were loaded into a 1\% certified low melt agarose gel (Bio-Rad, 321 1613112) in 0.5X TAE buffer with ladders (CHEF DNA Size Marker, 0.2-2.2 Mb, S. 322 cerevisiae Ladder: Bio-Rad, 1703605; CHEF DNA Size Marker, 1-3.1 Mb, H. wingei 323 Ladder: Bio-Rad, 1703667) and PFGE was performed using the CHEF Mapper XA 324 System (Bio-Rad) according to the manufacturer's instructions and using the following 325 settings: $0.5 \mathrm{X}$ TAE running buffer, $14^{\circ} \mathrm{C}$, two-state mode, run time duration of 16 hours 32639 minutes, initial switch time of 20.16 seconds, final switch time of 2 minutes 55.12 327 seconds, gradient of $6 \mathrm{~V} / \mathrm{cm}$, included angle of $120^{\circ}$, and linear ramping. Gel was stained 328 with $3 X$ Gelred (Biotium) with $0.1 \mathrm{M} \mathrm{NaCl}$ on a rocker for 30 minutes covered from light 329 and imaged. Bands were then extracted and DNA was purified from agarose blocks using 330 beta-Agarase I (New England BioLabs, M0392L) following the manufacturer's 331 instructions. 
334 To perform short-read sequencing on DNA isolated by CRISPR-CATCH, we first 335 transposed it with $\mathrm{Tn} 5$ transposase produced as previously described ${ }^{28}$, in a 50 ul reaction 336 with TD buffer ${ }^{29}, 10 \mathrm{ng}$ DNA and $1 \mathrm{ul}$ transposase. The reaction was performed at $37^{\circ} \mathrm{C}$ 337 for 5 minutes, and transposed DNA was purified using MinElute PCR Purification Kit 338 (Qiagen, 28006). Libraries were generated by 7 rounds of PCR amplification using 339 NEBNext High-Fidelity 2X PCR Master Mix (NEB, M0541L), purified using SPRIselect 340 reagent kit (Beckman Coulter, B23317) with double size selection (0.8X right, 1.2X left) 341 and sequenced on the Illumina Miseq platform or on an Illumina NovaSeq 6000. For 342 GBM39 enrichment and mutation analyses in Figures 1 and 2, a 1.2X left-side selection 343 was performed using SPRIselect. Sequencing data were processed as described above 344 for WGS.

\section{Genetic Variant Analyses}

347 SNVs were identified using GATK (version 4.2.0.0) ${ }^{30}$ from short-read sequencing data as 348 follows. First, base quality score recalibration was performed on bam files (generated as 349 described above) using gatk BaseRecalibrator followed by gatk ApplyBQSR. Covariates 350 were analyzed using gatk AnalyzeCovariates. SNVs were called using gatk Mutect2 from 351 the recalibrated bam files, and SNVs were filtered using gatk FilterMutectCalls. Finally, 352 vcf files were converted to table format using gatk VariantsToTable with the following 353 parameters: "-F CHROM -F POS -F REF -F ALT -F QUAL -F TYPE -GF AD -GF GQ -GF

354 PL -GF GT". Mutation variant allele frequencies (VAFs) were calculated by dividing 355 alternate allele occurrences by the sum of reference and alternate allele occurrences. 356 SNVs which had coverage depth of 5 or less, had a VAF of 1, or were not detected in 357 WGS were filtered out. Read alignment was visualized using Gviz in R.

SVs from short-read sequencing were identified with DELLY ${ }^{31}$ (version 0.8.7; using Boost 360 version 1.74 .0 and HTSlib version 1.12) using the delly call command. BCF files were 361 converted to VCF using bcftools view in Samtools ${ }^{32}$. VAFs were calculated using both 362 imprecise and precise variants. Read alignment was visualized using Gviz in R. 
Hung et al., (CHANG), p.14

365 DNA isolated by CRISPR-CATCH was directly used without amplification for nanopore 366 sequencing. Sequencing libraries were prepared using the Rapid Sequencing Kit (Oxford 367 Nanopore Technologies, SQK-RAD004) according to the manufacturer's instructions. 368 Sequencing was performed on a MinION (Oxford Nanopore Technologies).

Bases were called from fast5 files using guppy (Oxford Nanopore Technologies, version 5.0.16) within Megalodon (version 2.3.3) and DNA methylation status was determined using Rerio basecalling models with the configuration file "res_dna_r941_min_modbases-all-context_v001.cfg" and the following parameters: “-outputs basecalls mod_basecalls mappings mod_mappings mods per_read_mods -mod-motif Z CG 0 --write-mods-text --mod-output-formats bedmethyl wiggle --mod-mapemulate-bisulfite --mod-map-base-conv C T --mod-map-base-conv Z C". Methylation calls on single molecules were visualized using Integrative Genome Viewer (IGV, version 2.11.1) in bisulfite mode.

To quantify $5 \mathrm{mC}-\mathrm{CpG}$ methylation levels across an entire locus, rolling averages of $\mathrm{CpG}$ methylation percentages were calculated using a window of $100 \mathrm{bp}$ sliding every $10 \mathrm{bp}$ (unless otherwise specified). Rolling averages of ecDNA and the native chromosomal locus were linearly regressed using the $\mathrm{Im}$ function in $\mathrm{R}$. Standardized residual for the linear regression for each window was calculated using the rstandard function to represent relative methylation frequencies on ecDNA compared to chromosomal DNA. To identify accessible regions which are differentially methylated on ecDNA, we first filtered on ATAC-seq peaks which had log-normalized coverage above 9 (calculated by

388 DESeq2 as described in the ATAC-seq section below; normalized coverage for each peak was divided by peak width after adding 1, scaled to 500 and log2-transformed). Next, 390 methylation sites with coverage above 5 for both the purified ecDNA and chromosomal 391 locus and overlapping filtered ATAC-seq peaks were linearly regressed using the Im 392 function in R. Standardized residual for the linear regression for each CpG site was 393 calculated using the rstandard function. For each ATAC-seq peak, a $z$ score was 394 calculated using the formula $z=(x-m) / S$.E., where $x$ is the mean $\mathrm{CpG}$ residual within the 395 peak, $m$ is the mean residual of all $\mathrm{CpG}$ sites, and S.E. is the standard error calculated 
396 from the standard deviation of all CpG sites divided by the square root of the number of

397 CpG sites within the peak. $Z$ scores were used to compute two-sided $p$ values using the 398 normal distribution function, which were adjusted with p.adjust in $R$ (version 3.6.1) using 399 the Benjamini-Hochberg Procedure.

401 To quantify co-occurrence of methylated or unmethylated $\mathrm{CpGs}$ on single molecules, 402 methylation calls on the "+" strand were offset by 1 bp to match the locations of the 403 corresponding CpG sites on the "-" strand. CpG sites where the base probabilities of 404 methylation were above 0.7 were categorized as methylated, and sites where the base 405 probabilities of unmodified $\mathrm{CpG}$ were above 0.7 were categorized as unmethylated. For 406 each pair of $\mathrm{CpG}$ sites, co-occurrence was calculated by number of co-occurrences of 407 methylated or unmethylated $\mathrm{CpGs}$ on the same nanopore sequencing reads divided by 408 total number of occurrences in which the two CpG sites can be successfully categorized 409 as either methylated or unmethylated.

\section{ATAC-seq}

412 ATAC-seq data for GBM39 were generated by a previously published study ${ }^{8}$ and raw 413 fastq reads obtained from the NCBI Sequence Read Archive, under BioProject accession 414 PRJNA506071. Adapter-trimmed reads were aligned to the hg19 genome using Bowtie2 415 (2.1.0). Aligned reads were filtered for quality using samtools (version 1.9) ${ }^{32}$, duplicate 416 fragments were removed using Picard's MarkDuplicates (version 2.25.3), and peaks were 417 called using MACS2 (version 2.2.7.1) ${ }^{33}$ with a q-value cut-off of 0.01 and with a no-shift 418 model. Peaks from replicates were merged, read counts were obtained using bedtools 419 (version 2.30.0) ${ }^{34}$ and normalized using DESeq2 (using the "counts" function in DESeq2 $420 \quad$ with normalized $=$ TRUE; version 1.26.0 $)^{35}$.

\section{MNase-seq}

423 MNase-seq data for GBM39 were generated by a previously published study ${ }^{8}$ and raw 424 fastq reads obtained from the NCBI Sequence Read Archive, under BioProject accession 425 PRJNA506071. Reads were trimmed of adapter content with Trimmomatic ${ }^{25}$ (version 426 0.39), aligned to the hg19 genome using BWA MEM ${ }^{26}$ (0.7.17-r1188), and PCR duplicates 
427 removed using Picard's MarkDuplicates (version 2.25.3). Coverage of nucleosome 428 midpoints was obtained using bamCoverage from deepTools (version 3.5.1) with the 429 following parameters: “--MNase --binSize 1".

\section{De Novo Amplicon Reconstruction}

432 Using short-read sequencing data from CRISPR-CATCH with double size selection as 433 described above, we implemented new strategies and modified existing methods ${ }^{6}$ to 434 resolve ecDNA structures. Broadly, the methods involved seven steps. The last six steps 435 are available in a CRISPR-CATCH reconstruction pipeline, available at 436 https://github.com/siavashre/CRISPRCATCH.

438 1. To identify the regions of interest, we ran PrepareAA 439 (https://github.com/lluebeck/PrepareAA) (version 0.931.4) and AmpliconArchitect 440 (version 1.2_r2, available from https://github.com/iluebeck/AmpliconArchitect) on two 441 public bulk SNU16 WGS datasets (SRX546661236; SRR530826, Genome Research 442 Foundation) and found comparable graphs in both. We used PrepareAA with BWA$443 \mathrm{MEM}^{26}$ (version 0.7.12-r1039) to align reads to $\mathrm{hg} 19$ and $\mathrm{CNVKit}^{37}$ (version 0.9.7) to 444 generate seed regions having copy number $(C N)>5$. These regions were provided to 445 AA, which constructed a $\mathrm{CN}$-aware breakpoint graph. The genome regions $\mathrm{AA}$ included 446 in the graph were converted to bed format and used as the seed regions in the analysis 447 of each PFGE band, so that the regions studied were always consistent between bands.

449 2. Using WGS reads generated from CRISPR-CATCH-purified DNA, for each band we 450 next aligned to the hy19 reference genome using PrepareAA which included BWA MEM 451 and a PCR-duplicate removal step (using samtools ${ }^{32}$ version 1.3.1), and we also made 452 estimates of insert size distribution using Picard (version 2.25.6) for quality control 453 purposes.

455 3. The aligned PFGE data and seed regions identified from bulk sequencing were 456 provided to AmpliconArchitect (version 1.2_r2) to construct the $\mathrm{CN}$-aware breakpoint 457 graph, using non-default arguments -downsample -1 -pair_support 2 -no_cstats - 
458 insert_sdevs 8.5 . The -insert_sdevs parameter allows for larger insert size variation 459 without forming breakpoints from read pairs marked as discordant, as we found high 460 insert size variance occurred frequently in DNA extracted from the gels. Following AA, we 461 ran a script on the resulting $\mathrm{CN}$-aware breakpoint graph to filter non-foldback graph edges 462 joining regions smaller than $1 \mathrm{kbp}$ from the graph, representing potential unfiltered artifact 463 edges arising from overdispersion in insert size variance, in order to reduce the 464 complexity of the graph when performing pathfinding. Since the edges removed joined 465 regions not more than $1 \mathrm{kbp}$ apart and did not lead to changes in the orientation of the 466 genome, this step had a negligible effect on the resulting paths. This utility for filtering AA 467 graphs is made available as part of PrepareAA (graph_cleaner.py).

469 4. Central to the method for ecDNA reconstruction is the assumption that a single ecDNA 470 is being analyzed within the graph, and as a result the estimated genomic copy numbers 471 should closely relate to the number of times a segment appears within the ecDNA. We 472 termed the number of times a segment appeared within a single ecDNA as the 473 "multiplicity" of a genomic segment. The path finding method first removes low CN 474 elements from the graph representing the background genome and contamination from 475 incomplete separation of ecDNAs (i.e., remove segments with $\mathrm{CN}$ below $20 \%$ of the 476 maximum $\mathrm{CN}$ of all segments having length $>100 \mathrm{bp}$, or below $10 \%$ of the maximum, if 477 the maximum $\mathrm{CN}$ is $>10000$ ). In the remaining segments, we assumed that the majority 478 of segments appeared once within an ecDNA. We assumed that ecDNA where the 479 majority of segments are present more than once would reflect cases where two or more 480 ecDNA were present instead of one. Thus, to compute the multiplicity of each graph 481 segment, the method computes the $40^{\text {th }}$ percentile of the remaining graph segment copy 482 numbers and assigns that copy number, $S_{1}$, to multiplicity $=1$. For each segment, $i$, in the 483 graph, we computed its multiplicity, $M(i)$ as.

$$
M(i)=\operatorname{round}\left(\frac{C N(i)}{S_{1}}\right)
$$
5. To find paths in the graph which represented candidate ecDNA structures, we used an 
488

estimated maximum molecule size suggested by the CRISPR-CATCH data. Candidate ecDNA structures are determined through a constrained depth-first search (DFS) approach, which attempts to identify paths in the graph, and performs the process starting at every segment in the graph assigned a non-zero multiplicity. During the search, the length of the path (in base pairs) must remain less than the maximum allowed length $(L)$. For every segment $i$, appearing $n_{i}$ times in the path, $n_{i} \leq M(i)$. The DFS recursion terminates if either constraint is violated, and the current path is scored as $\sum_{i} n_{i}$. The path is compared against the current best path (initiated as an empty path with score 0 ) and updated if it scores higher. Both the best-scoring cyclic paths as well as the best-scoring paths regardless of cyclic status are returned after removing all duplicate (identical) paths from the collection of best-scoring paths. This utility is also individually available from PrepareAA (plausible_paths.py).

6. We found a number of features of both the breakpoint graph and the reconstructions to be informative about the quality of the data in the band. We developed quality annotations reported along with each reconstruction to provide users with annotations about the confidence of the reconstruction. We note that $\mathrm{CN}$-aware breakpoint graphs derived from NGS data may contain a number of error sources including missing edges between graph segments and incorrect estimation of copy numbers (leading sometimes to incorrect estimation of multiplicity). The method applies the following filters.

a) In the amplicon region analyzed by $A A$, the total amount of amplified material (nonzero multiplicity) should not significantly exceed the maximum estimated molecular size of the band (if provided). We used a cutoff such that amplicons with $1.4 \mathrm{x}$ the maximum estimated molecular size of the band were flagged for low quality (incomplete separation of ecDNA).

b) Changes in multiplicity must be accompanied by one or more breakpoint junctions, and thus for a breakpoint graph with $|e|$ total edges, amplicons where

$$
\frac{|e|}{\max (M(i))}<1
$$


518 were flagged for low quality (missing graph edges).

c) We defined a root mean square residual for the unexplained copy numbers of $M(i)$. In a given path, for each segment $i$, having $n_{i}$ occurrences in the path, the root mean square

522 residual was defined as

$$
R M S R=\sqrt{\frac{1}{N} \sum_{i=1}^{N}\left(n_{i}-M(i)\right)^{2}}
$$

524 where $N$ is the number of segments having non-zero multiplicity in the graph. We set a 525 default cutoff such that amplicons with RMSR $>0.9$ were flagged as low quality (too many 526 amplified graph segments having incompletely used multiplicity).

d) To assess how tightly segment copy numbers could be segregated by segment multiplicity, we computed the Davies-Bouldin index ${ }^{38}$ (DBI) on the clusters of copy numbers. Each cluster was comprised of all segment copy numbers assigned to a multiplicity (singleton clusters excluded), and the centroid of the cluster was the mean $\mathrm{CN}$

532 for the cluster. Amplicons where the DBI was $>0.3$ were flagged as low quality due to noisy copy number estimation.

e) If a minimum molecular size for the band was given, we flagged reconstructions which fell below that $90 \%$ of that value as low quality as they reflected incomplete reconstructions.

539 f) If no segment in the reconstruction overlapped the CRISPR-Cas9 target site, we

540 flagged it as being low quality as it was either an incomplete reconstruction, or the 541 incorrect amplicon was detected.

543 7. Since the reconstructed paths are reported in the textual AA_cycles.txt format, the 544 method also provides automated circular visualizations of the structures and the WGS 545 coverage tracks which are generated by CycleViz (https://github.com/jluebeck/CycleViz) 546 (version 0.1.0). 
Hung et al., (CHANG), p20

\section{$548 \quad$ Optical Mapping}

549 Optical maps from SNU16 cells were generated as follows: ultra-high molecular weight

550 (UHMW) DNA was extracted from 1.5 million frozen cells preserved in DMSO following 551 the manufacturer's instructions (Bionano Genomics, \#30398, 80042). Briefly, cells were 552 digested with Proteinase K (Puregene \#158920) and RNAse A (Puregene \#158922) and 553 then the DNA was precipitated with isopropanol and bound with nanobind magnetic disks.

554 Bound UHMW DNA was resuspended in elution buffer (EB) and quantified with Qubit 555 dsDNA BR assay kit (ThermoFisher Scientific, Q32850). The final DNA concentration was

556 initially too high $(280 \mathrm{ng} / \mu \mathrm{l})$, therefore UHMW DNA was further diluted with EB, gently 557 mixed with a wide-bore tip five times, and allowed to relax at room temperature for two 558 days. Upon resting, UHMW DNA was diluted to $110 \mathrm{ng} / \mu \mathrm{l}$ in EB and DNA labeling was 559 performed following the manufacturer's instructions (Bionano Genomics, \#30206, 80005). 560 Standard Direct Labeling Enzyme 1 (DLE-1) reactions were carried out using $750 \mathrm{ng}$ of 561 purified UHMW DNA. Using the Qubit dsDNA HS assay kit (ThermoFisher Scientific, 562 \#32854), the final labeled DNA concentration was determined as $5.4 \mathrm{ng} / \mu \mathrm{l}$ with a 563 coefficient of variation of 0.026 . The fluorescently labeled DNA molecules were loaded 564 onto the Saphyr Chip G2.3 (Bionano Genomics, \#20366, 30142) and were imaged 565 sequentially across nanochannels on a Saphyr instrument (Bionano Genomics, \#90023). 566 An effective genome coverage of approximately 340X, using molecules >= $150 \mathrm{kbp}$ 567 (molecule N50 of $0.2505 \mathrm{Mbp}$ ) was achieved.

De novo assembly of SNU16 was performed with Bionano's de novo assembly pipeline 570 (Bionano Solve v3.6, \#90023) using standard haplotype aware arguments. With the 571 Overlap-Layout-Consensus paradigm, pairwise comparison of DNA molecules of 572 approximately $130 \mathrm{X}$ coverage was used to create a layout overlap graph, which was then 573 used to generate the initial consensus genome maps, which had a contig N50 of 50 Mbp.

574 By realigning molecules to the genome maps ( $P$ value cutoff of $<10^{-12}$ ), and by using only 575 the best matched molecules, a refinement step was done to refine the label positions on 576 the genome maps and to remove chimeric joins. Next, during an extension step, the 577 software aligned molecules to genome maps $\left(P<10^{-12}\right)$, and extended the maps based on 
578 the molecules aligning past the map ends. Overlapping genome maps were then merged

$579\left(\mathrm{P}<10^{-16}\right)$. These extension and merge steps were repeated five times before a final 580 refinement $\left(\mathrm{P}<10^{-12}\right)$ was applied to "finish" all genome maps.

\section{Validating candidate structures with optical mapping}

583 To validate candidate ecDNA paths we used long-range optical mapping (OM) data.

584 Previously, we developed a method, AmpliconReconstructor $(A R)^{7}$, which uses OM data 585 and AA's outputs as inputs. AR attempts to identify paths within the breakpoint graph 586 supported by OM contigs. In the CRISPR-CATCH data, the graphs may have many 587 smaller segments than the graphs derived from bulk WGS, due to noisier CN profiles in 588 the gel-extracted DNA, or due to highly complex ecDNA structures having very dense 589 breakpoints. In these cases, large numbers of graph segments may be too small to 590 reliably align against OM contigs using AR's standard approach. Thus, we added a 591 method to AR whereby the user can provide an AA-formatted cycles.txt file containing the 592 full candidate path. The individual segments are combined into a single long genome 593 sequence, then converted to an in silico digested OM sequence. The combined candidate 594 OM sequence is then aligned with OM contigs using AR's SegAligner method. The 595 resulting candidate sequence alignment label indices are mapped back to the original 596 uncombined candidate path. Contig alignments for the candidate path were combined if 597 necessary and visualized using CycleViz. The candidate path alignment utility added to 598 AR for this analysis is available at https://github.com/jluebeck/AmpliconReconstructor.

\section{Data Availability}

601 Sequencing data generated in this study are deposited in SRA under BioProject accession PRJNA777710.

\section{Code Availability}

605 Custom code to perform reconstructions of candidate ecDNA structures from CRISPR$606 \mathrm{CATCH}$ data is available at https://github.com/siavashre/CRISPRCATCH.

\section{Acknowledgements}


609 We thank members of the Chang and Bafna laboratories for discussions. H.Y.C. was 610 supported by NIH R35-CA209919 and RM1-HG007735. K.L.H. was supported by a

611 Stanford Graduate Fellowship. H.Y.C. is an Investigator of the Howard Hughes Medical 612 Institute.

\section{Author Contributions}

615 K.L.H. and H.Y.C. conceived the project. K.L.H. performed experiments for CRISPR616 CATCH method development for ecDNA purification and analyses of genetic variants and 617 epigenetic features from short-read sequencing and nanopore sequencing data. J.L. and 618 S.R.D. analyzed short-read sequencing and optical mapping data for amplicon 619 reconstruction. C.C. and J.A.L. generated optical mapping data and provided de novo 620 assembly and rare variant analysis results. W.J.G. advised on single molecule 621 sequencing. P.M., V.B. and H.Y.C. guided data analysis and provided feedback on 622 experimental design. K.L.H. and H.Y.C. wrote the manuscript with input from all authors.

\section{Competing Interests}

625 H.Y.C. is a co-founder of Accent Therapeutics, Boundless Bio, Cartography Biosciences, 626 and an advisor of 10x Genomics, Arsenal Biosciences, and Spring Discovery. P.M. and 627 V.B. are co-founders and advisors of Boundless Bio.

\section{Materials \& Correspondence}

630 Correspondence and requests for materials should be addressed to Howard Y. Chang 631 (howchang@stanford.edu). 
Hung et al., (CHANG), p23

\section{3}

634

635

636

637

638

639

640

641

642

643

644

645

646

647

648

649

650

651

652

653

654

655

656

657

658

659

660

661

662

663

664

665

666

667

668

669

670

671

672

673

674

675

676

677

\section{REFERENCES}

1. Turner, K. M. et al. Extrachromosomal oncogene amplification drives tumour evolution and genetic heterogeneity. Nature 543, 122-125 (2017).

2. Kim, $\mathrm{H}$. et al. Extrachromosomal DNA is associated with oncogene amplification and poor outcome across multiple cancers. Nature Genetics 52, 891-897 (2020).

3. Verhaak, R. G. W., Bafna, V. \& Mischel, P. S. Extrachromosomal oncogene amplification in tumour pathogenesis and evolution. Nature Reviews Cancer 19, 283 (2019).

4. Møller, H. D. et al. Circular DNA elements of chromosomal origin are common in healthy human somatic tissue. Nature Communications 9, 1069 (2018).

5. Wang, Y. et al. eccDNAs are apoptotic products with high innate immunostimulatory activity. Nature 1-7 (2021) doi:10.1038/s41586-021-04009-w.

6. Deshpande, V. et al. Exploring the landscape of focal amplifications in cancer using AmpliconArchitect. Nature Communications 10, 392 (2019).

7. Luebeck, J. et al. AmpliconReconstructor integrates NGS and optical mapping to resolve the complex structures of focal amplifications. Nature Communications 11, 4374 (2020).

8. Wu, S. et al. Circular ecDNA promotes accessible chromatin and high oncogene expression. Nature 1-5 (2019) doi:10.1038/s41586-019-1763-5.

9. Møller, H. D., Parsons, L., Jørgensen, T. S., Botstein, D. \& Regenberg, B. Extrachromosomal circular DNA is common in yeast. PNAS 112, E3114-E3122 (2015).

10. Koche, R. P. et al. Extrachromosomal circular DNA drives oncogenic genome remodeling in neuroblastoma. Nature Genetics 1-6 (2019) doi:10.1038/s41588-0190547-z.

11. van der Bliek, A. M., Lincke, C. R. \& Borst, P. Circular DNA of 3T6R50 double minute chromosomes. Nucleic Acids Research 16, 4841-4851 (1988).

12. Borst, P., Van Der Bliek, A. M., Van Der Velde-Koerts, T. \& Hes, E. Structure of amplified DNA, analyzed by pulsed field gradient gel electrophoresis. Journal of Cellular Biochemistry 34, 247-258 (1987).

13. Nassonova, E. S. Pulsed field gel electrophoresis: Theory, instruments and application. Cell Tiss. Biol. 2, 557 (2008).

14. Jiang, W. et al. Cas9-Assisted Targeting of CHromosome segments CATCH enables one-step targeted cloning of large gene clusters. Nature Communications $\mathbf{6}$, $1-8$ (2015).

15. Gabrieli, T. et al. Selective nanopore sequencing of human BRCA1 by Cas9assisted targeting of chromosome segments (CATCH). Nucleic Acids Res 46, e87e87 (2018).

16. Sarkaria, J. N. et al. Identification of molecular characteristics correlated with glioblastoma sensitivity to EGFR kinase inhibition through use of an intracranial xenograft test panel. Mol Cancer Ther 6, 1167-1174 (2007).

17. Nathanson, D. A. et al. Targeted Therapy Resistance Mediated by Dynamic Regulation of Extrachromosomal Mutant EGFR DNA. Science 343, 72-76 (2014).

18. Nikolaev, S. et al. Extrachromosomal driver mutations in glioblastoma and low-grade glioma. Nature Communications 5, 5690 (2014). 
19. Lövkvist, C., Sneppen, K. \& Haerter, J. O. Exploring the Link between Nucleosome Occupancy and DNA Methylation. Frontiers in Genetics 8, 232 (2018).

20. Kelly, T. K. et al. Genome-wide mapping of nucleosome positioning and DNA methylation within individual DNA molecules. Genome Res. 22, 2497-2506 (2012).

21. Hung, K. L. et al. EcDNA hubs drive cooperative intermolecular oncogene expression. http://biorxiv.org/lookup/doi/10.1101/2020.11.19.390278 (2020) doi:10.1101/2020.11.19.390278.

22. Müller, C. A. et al. Capturing the dynamics of genome replication on individual ultralong nanopore sequence reads. Nature Methods 16, 429-436 (2019).

23. Shipony, Z. et al. Long-range single-molecule mapping of chromatin accessibility in eukaryotes. Nature Methods 1-9 (2020) doi:10.1038/s41592-019-0730-2.

24. Stergachis, A. B., Debo, B. M., Haugen, E., Churchman, L. S. \& Stamatoyannopoulos, J. A. Single-molecule regulatory architectures captured by chromatin fiber sequencing. Science 368, 1449-1454 (2020).

25. Bolger, A. M., Lohse, M. \& Usadel, B. Trimmomatic: a flexible trimmer for Illumina sequence data. Bioinformatics 30, 2114 (2014).

26. Li, H. \& Durbin, R. Fast and accurate short read alignment with Burrows-Wheeler transform. Bioinformatics 25, 1754-1760 (2009).

27. Overhauser, J. Encapsulation of Cells in Agarose Beads. in Pulsed-Field Gel Electrophoresis: Protocols, Methods, and Theories (eds. Burmeister, M. \& Ulanovsky, L.) 129-134 (Humana Press, 1992). doi:10.1385/0-89603-229-9:129.

28. Picelli, S. et al. Tn5 transposase and tagmentation procedures for massively scaled sequencing projects. Genome Res. 24, 2033-2040 (2014).

29. Corces, M. R. et al. An improved ATAC-seq protocol reduces background and enables interrogation of frozen tissues. Nature Methods 14, 959-962 (2017).

30. McKenna, A. et al. The Genome Analysis Toolkit: A MapReduce framework for analyzing next-generation DNA sequencing data. Genome Res. 20, 1297-1303 (2010).

31. Rausch, T. et al. DELLY: structural variant discovery by integrated paired-end and split-read analysis. Bioinformatics 28, i333-i339 (2012).

32. Li, H. et al. The Sequence Alignment/Map format and SAMtools. Bioinformatics 25, 2078-2079 (2009).

33. Zhang, Y. et al. Model-based Analysis of ChIP-Seq (MACS). Genome Biology 9, R137 (2008).

34. Quinlan, A. R. \& Hall, I. M. BEDTools: a flexible suite of utilities for comparing genomic features. Bioinformatics 26, 841-842 (2010).

35. Love, M. I., Huber, W. \& Anders, S. Moderated estimation of fold change and dispersion for RNA-seq data with DESeq2. bioRxiv (2014) doi:10.1101/002832.

36. Ghandi, M. et al. Next-generation characterization of the Cancer Cell Line Encyclopedia. Nature 569, 503-508 (2019).

37. Talevich, E., Shain, A. H., Botton, T. \& Bastian, B. C. CNVkit: Genome-Wide Copy Number Detection and Visualization from Targeted DNA Sequencing. PLOS Computational Biology 12, e1004873 (2016).

38. Davies, D. L. \& Bouldin, D. W. A cluster separation measure. IEEE Trans Pattern Anal Mach Intell 1, 224-227 (1979). 
a

b
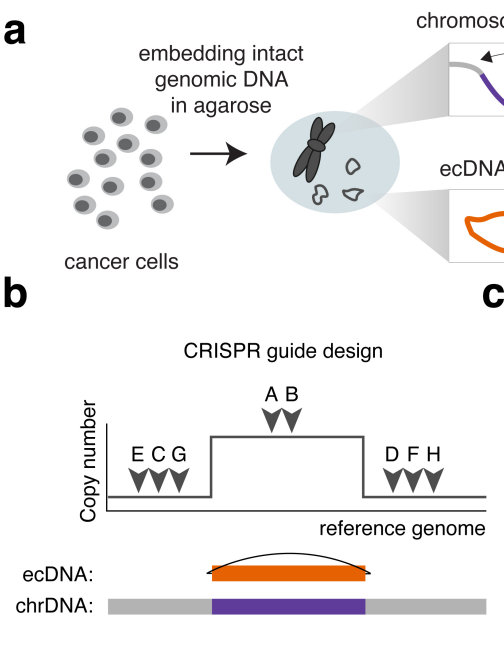

d

e
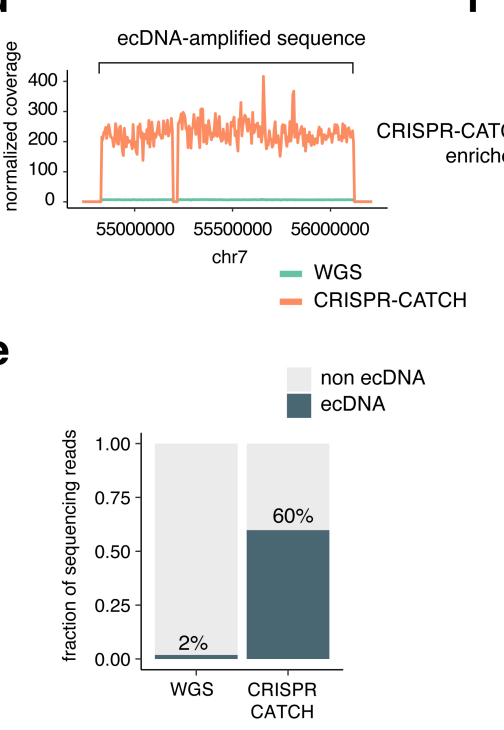
targets.

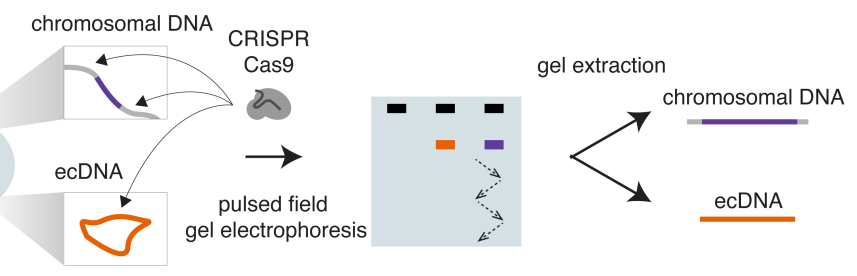

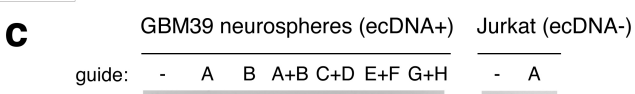

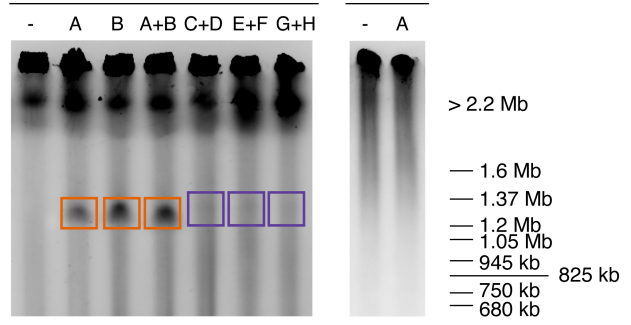

predicted sizes: $1.258 \mathrm{Mb} 1.233 \mathrm{Mb} \quad 1.258-1.293 \mathrm{Mb}$

f

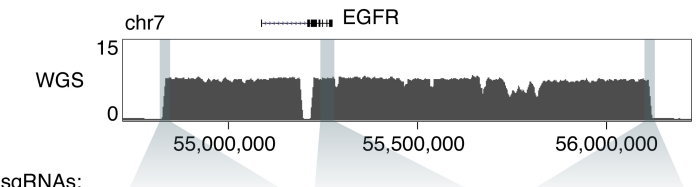

竞

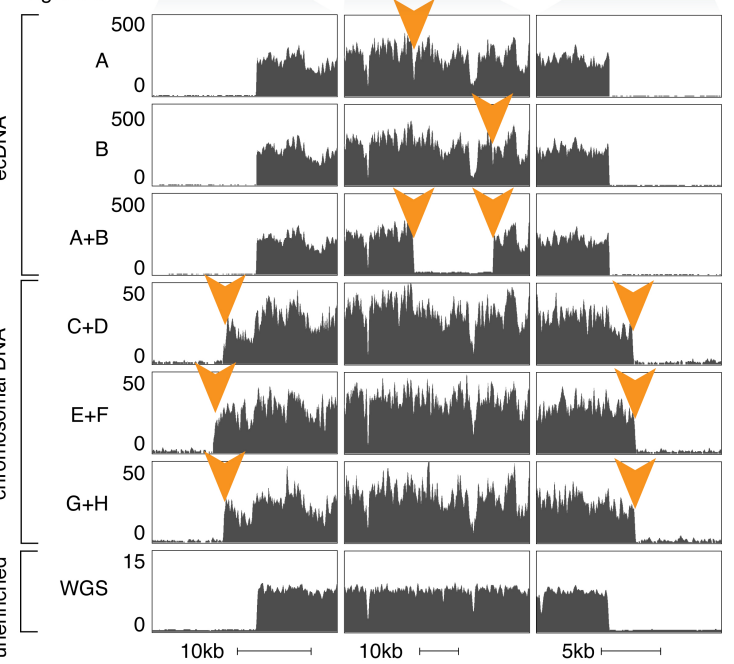

Figure 1. Isolation of megabase-sized ecDNA and its native chromosomal locus from the same cancer cell sample by CRISPR-CATCH. (a) Experimental workflow for purification of ecDNA and its corresponding chromosomal locus from the same cell sample. (b) Design of CRISPR sgRNAs for linearizing ecDNA circles or extracting the native chromosomal locus. (c) Representative PFGE images showing linearized ecDNA molecules and the chromosomal locus after treatment with indicated guides (Methods, raw gel images in Supplementary Figure 1, guide sequences in Supplementary Table 1). Boxed regions indicate parts of the gel that were extracted for DNA isolation. (d) Normalized short-read sequencing coverage of the expected ecDNA locus in unenriched WGS or after CRISPR-CATCH (guide A). (e) Fraction of total sequencing reads aligning to the expected ecDNA locus in unenriched WGS or after CRISPR-CATCH (guide A). (f) Sequencing tracks showing coverages for purified ecDNA and its chromosomal locus at the zoomed-in locations compared to WGS. Orange arrows indicate locations of sgRNA 
Hung et al., (CHANG), p26

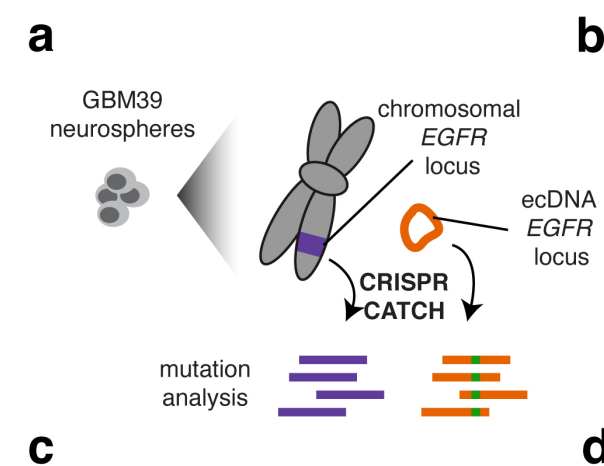

b
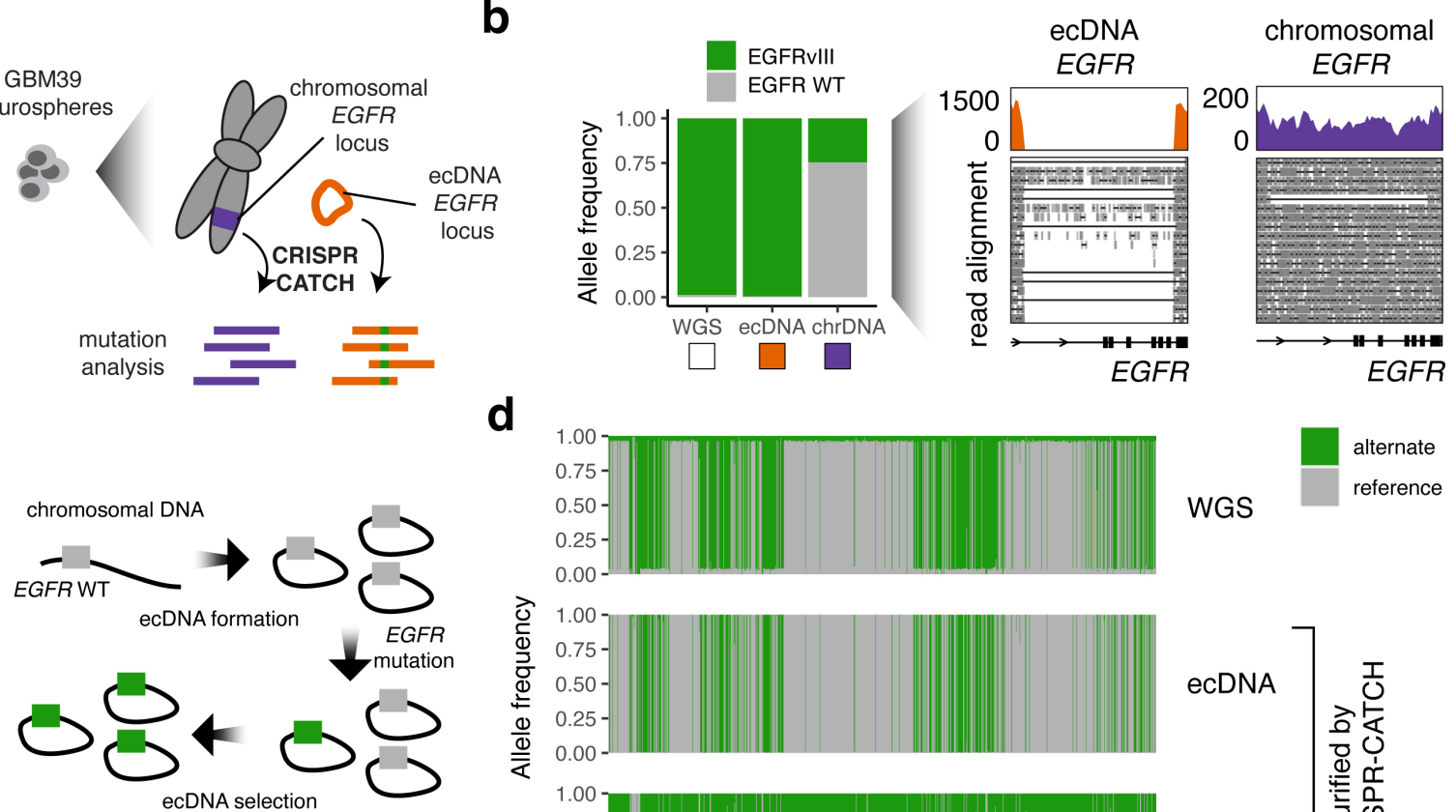

d

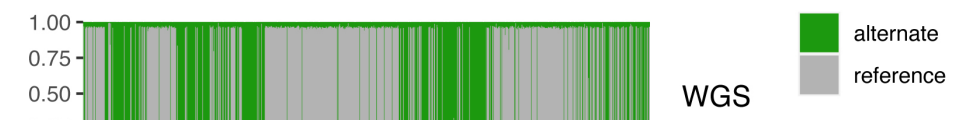

e
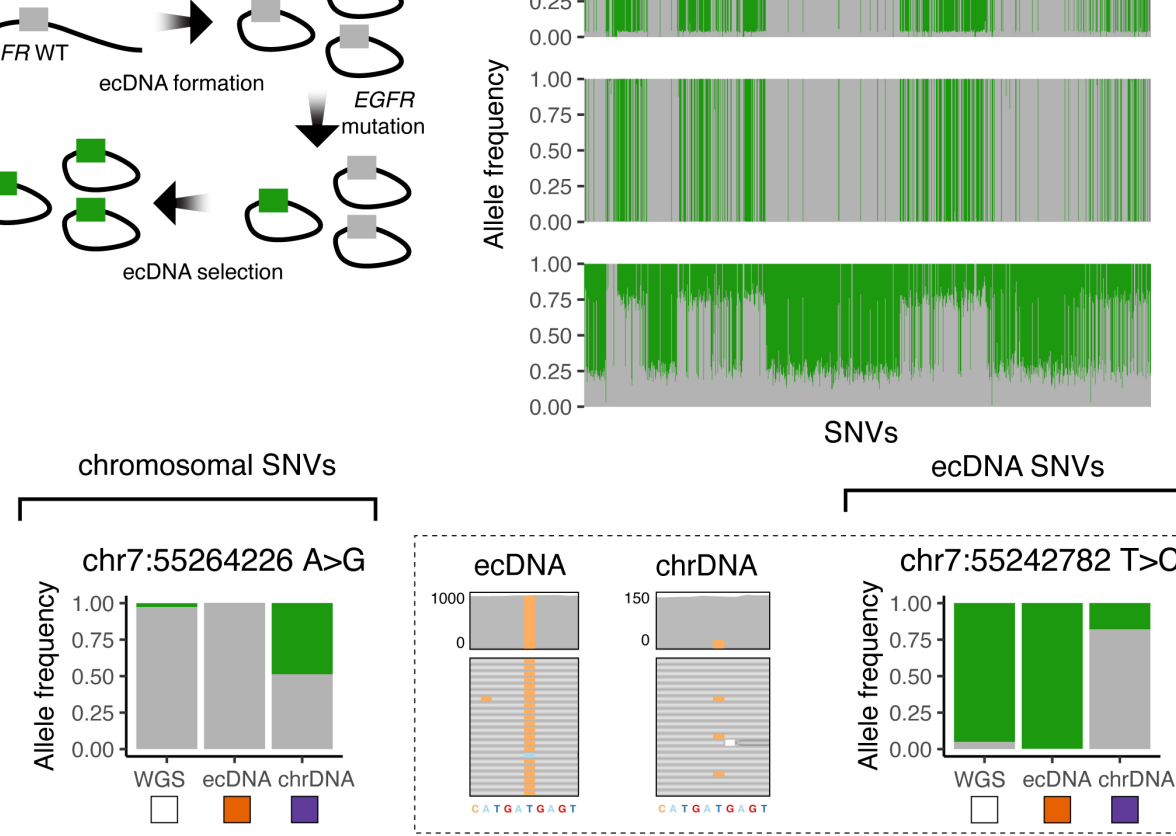
(a) Isolation of ecDNA (guide A) and the corresponding chromosomal locus (guides E+F) from GBM39 neurospheres by CRISPR-CATCH followed by mutation analysis using short-read sequencing. (b) Left: allele frequencies of the EGFRvIII mutant on ecDNA and chromosomal DNA. Right: sequencing coverage and junction reads supporting the EGFRvIII mutation and wild type. (c) EGFRvIII mutation likely occurred after ecDNA formation and became the predominant version of EGFR on ecDNA molecules as a result of selection. (d) Allele frequencies of SNVs identified in the ecDNA-amplified region and its native chromosomal locus. (e) Left and right: examples of ecDNA and chromosomal SNVs with various allele frequencies. Middle: sequencing reads supporting SNV identification. Each dashed box groups data for the same SNV. 
Figure 3. Comparison of CpG methylation statuses of ecDNA and its native chromosomal locus in the same cells. (a) Isolation of ecDNA (guide $A$ ) and the corresponding chromosomal locus (guides E+F) from GBM39 neurospheres by CRISPR$\mathrm{CATCH}$ followed by detection of $5 \mathrm{mC}-\mathrm{CpG}$ methylation by nanopore sequencing. (b) Negative correlation between mean methylation probabilities of ATAC-seq peaks and their ATAC-seq signals (Pearson's R, two-sided test). (c) Aggregated levels of relative CpG methylation of ecDNA compared to the chromosomal locus at top 50 ATAC-seq peaks in the ecDNA-amplified region. Mean methylation frequencies were calculated in 100-bp windows sliding every $10 \mathrm{bp}$. Relative frequencies were quantified from standardized residuals for a linear regression model for mean frequencies on ecDNA vs chromosomal DNA (Methods). (d) Bulk ATAC-seq track with differentially methylated regions annotated (Methods; $p$ values were Benjamini-Hochberg adjusted; regions with $p<0.005$ were considered significant). (e) Relative CpG methylation of ecDNA compared to the chromosomal locus in differential regions and concordance with accessibility by ATAC-seq and nucleosome positioning by MNase-seq. Mean methylation frequencies were calculated in 100-bp windows sliding every $10 \mathrm{bp}$. Relative frequencies were quantified from standardized residuals for a linear regression model for mean frequencies on ecDNA vs chromosomal DNA (Methods). (f) From top to bottom: Loess-smoothed methylation probability around the EGFR promoter; nanopore sequencing reads showing CpG methylation calls (grey denotes regions with no CpG sites); heatmap showing cooccurrence probabilities of unmethylated CpG sites on the same molecules; heatmap showing co-occurrence probabilities of methylated CpG sites on the same molecules (Methods). Raw PFGE image of purified DNA products is in Supplementary Figure 1. 
a

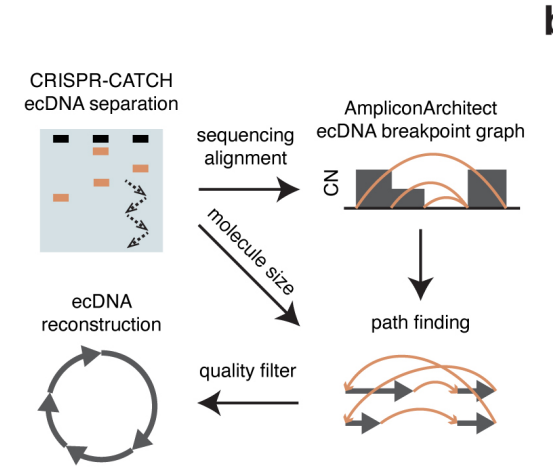

b

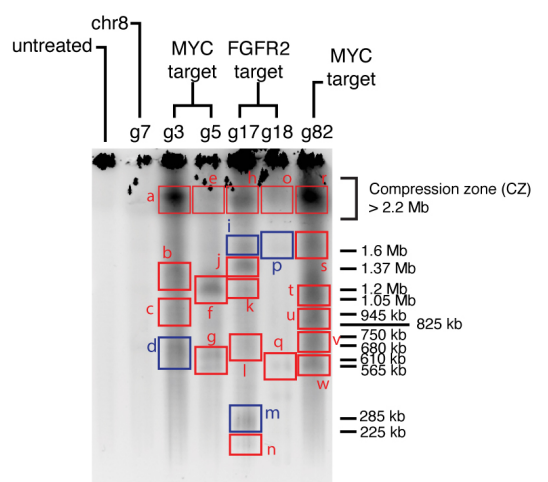

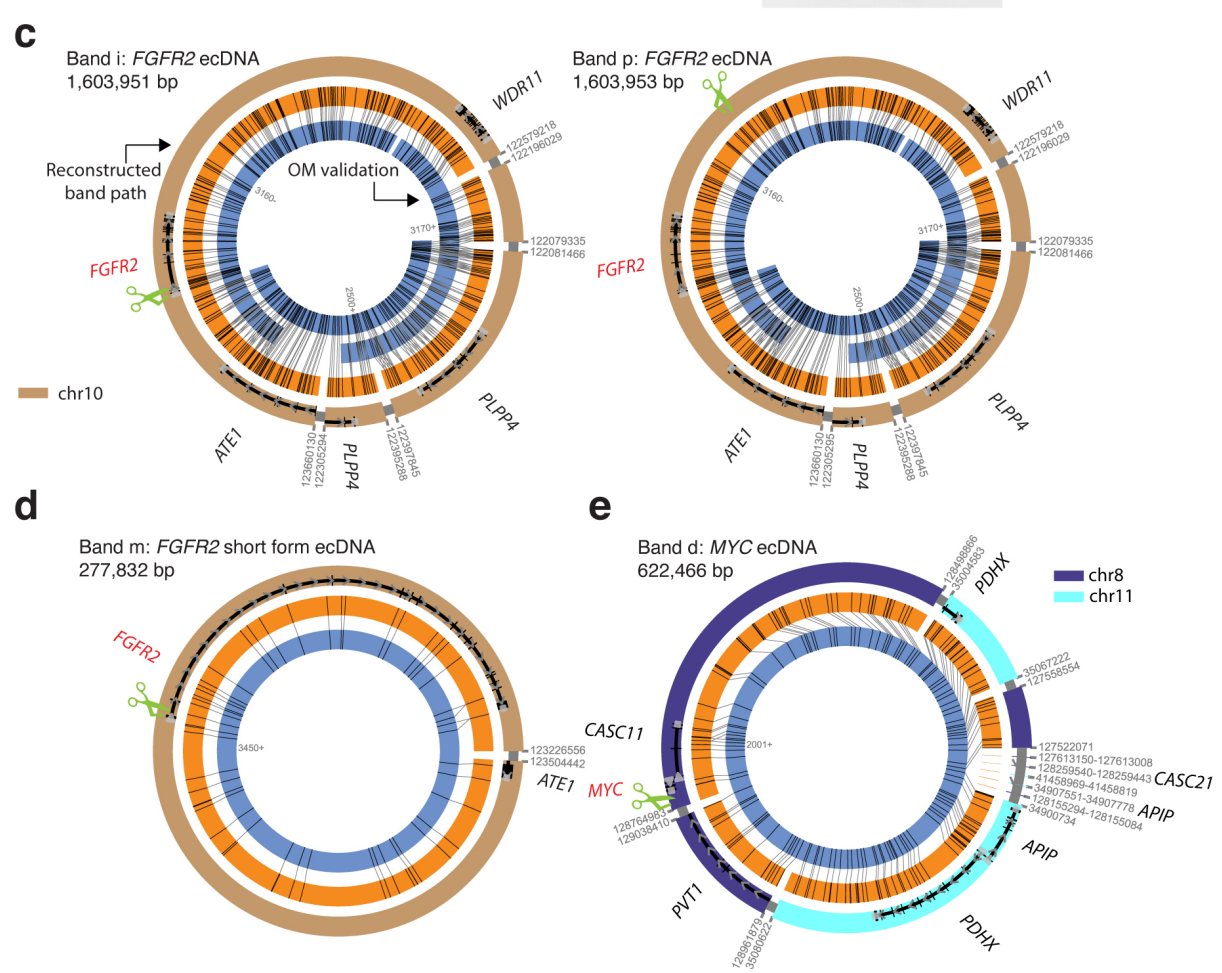

Figure 4. Reconstruction of multiple ecDNA species in the stomach cancer cell line SNU16. (a) Analysis of ecDNA structure using CRISPR-CATCH. ecDNA species are separated by size in PFGE and sequenced. AmpliconArchitect generates $\mathrm{CN}$-aware breakpoint graphs, which are used in combination with molecule sizes from PFGE to find paths and identify candidate ecDNA structures. (b) PFGE image for SNU16 after treatment with independent sgRNAs targeting either the FGFR2 or MYC locus (guide sequences in Supplementary Table 1, raw gel image in Supplementary Figure 1). Bands passing all quality filters are shown in blue. (c-e) ecDNA reconstructions using CRISPR-CATCH data (outer rings; thin grey bands mark connections between sequence segments). Optical mapping patterns (orange rings) and assembled contigs (blue rings, contig IDs indicated) validated CRISPR-CATCH reconstructions. Green scissors mark sgRNA target sites. Two equivalent FGFR2 ecDNA structures were reconstructed from bands " $\mathrm{i}$ " and " $p$ " from independent sgRNA treatments as shown in panel (c). A shortform FGFR2 ecDNA reconstructed from band " $\mathrm{m}$ " is shown in panel (d). A MYC ecDNA reconstructed from band "d" containing sequences from chromosomes 8 and 11 is shown in panel (e). 
Hung et al., (CHANG), p29

a

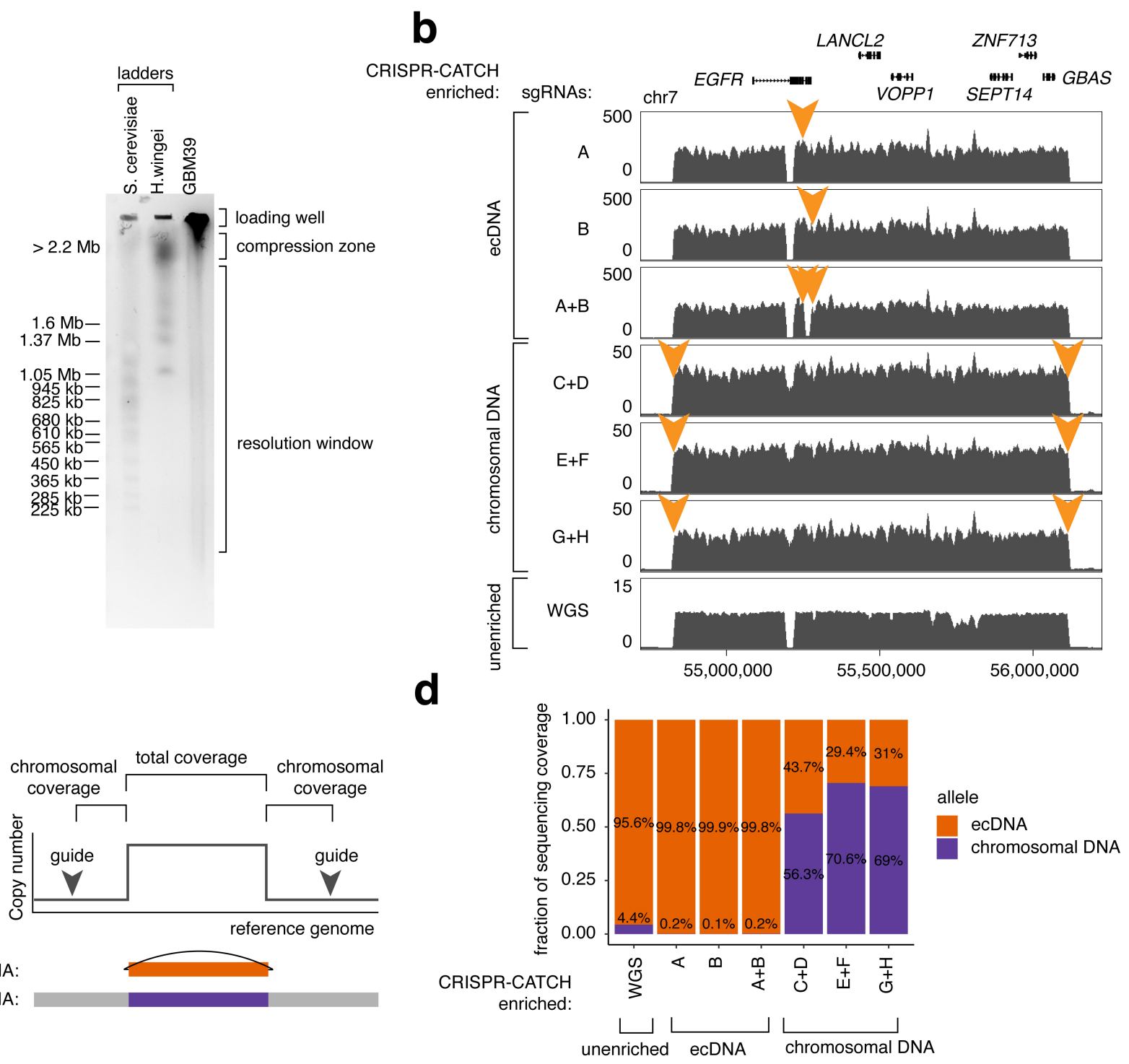

C
Extended Data Figure 1. Enrichment of circular ecDNA by CRISPR-CATCH. (a) A representative PFGE image showing size ladders and GBM39 ultra-high molecular weight (UHMW) genomic DNA without in-vitro CRISPR-Cas9 linearization. UHMW DNA was trapped in the loading well and the upper compression zone. (b) Full sequencing tracks showing coverage for purified ecDNA and its chromosomal locus at the EGFR amplified region compared to WGS. Zoomed-in tracks are shown in Figure 1f. Orange arrows indicate locations of sgRNA targets. (c) Chromosomal overhangs from chromosome-targeting guides (guides $\mathrm{C}-\mathrm{H}$ ) outside of the ecDNA-amplified region were used for calculating sequencing coverage of the chromosomal allele. The mean coverage of the 5' and 3' chromosomal overhangs was calculated. The coverage of ecDNA alleles was calculated by subtracting chromosomal coverage from total coverage in the ecDNAamplified region. (d) Relative sequencing coverage of chromosomal DNA and ecDNA alleles in WGS or CRISPR-CATCH samples. 
a

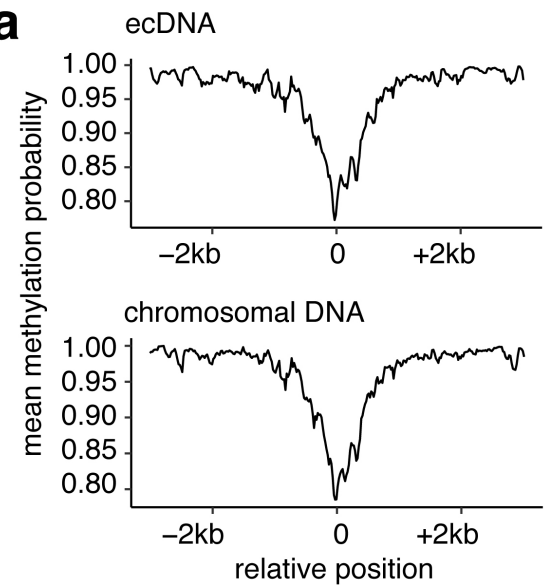

C

b

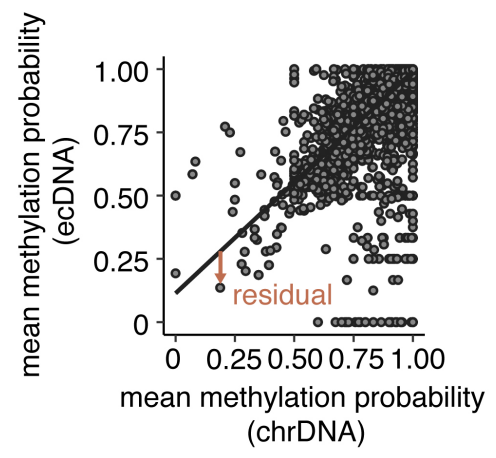

less methylated on:

chrDNA

ecDNA
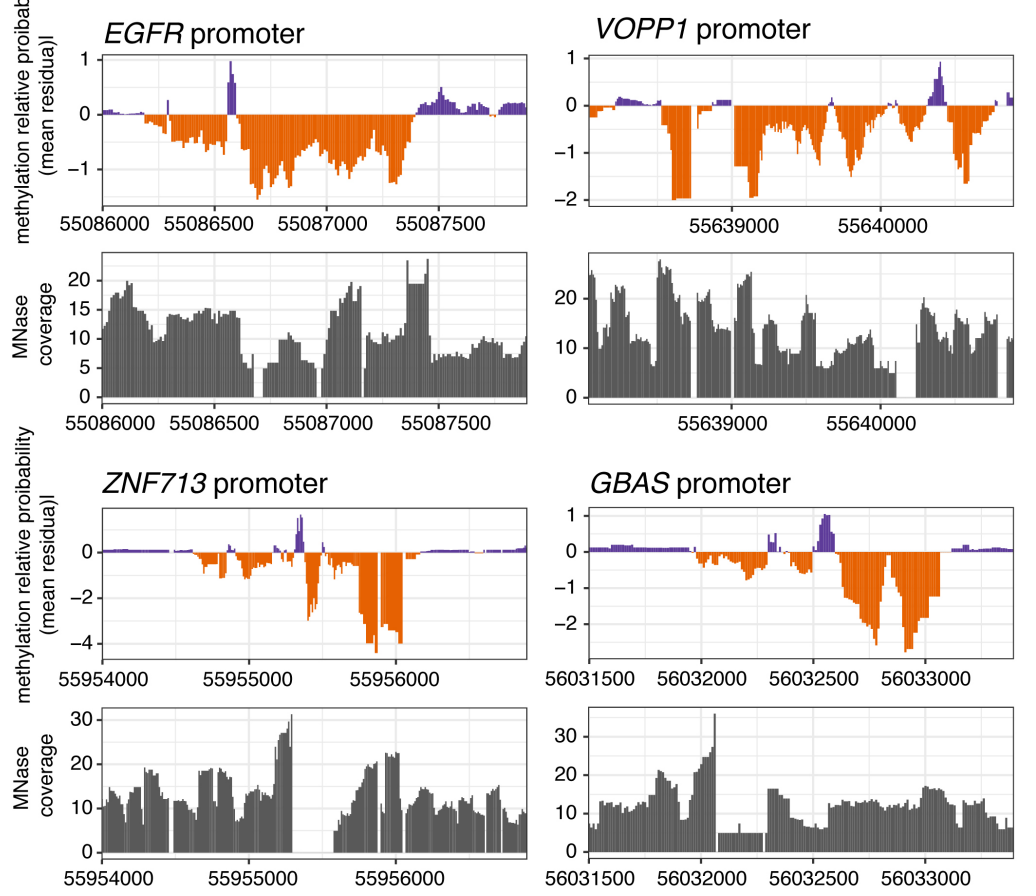

Extended Data Figure 2. Quantification of $5 \mathrm{mC}-\mathrm{CpG}$ methylation probability of ecDNA and the native chromosomal locus. (a) Aggregated CpG methylation probability of ecDNA and chromosomal DNA at the top 50 ATAC-seq peaks with highest coverage in the amplified region. Mean methylation frequencies were calculated in 100bp windows sliding every $10 \mathrm{bp}$. (b) Linear regression model of mean methylation probabilities of ecDNA vs chromosomal DNA. Mean methylation probabilities were calculated in 100-bp windows sliding every $10 \mathrm{bp}$ in the ecDNA-amplified region. Each point represents a window mean. Brown arrow demonstrates the standardized residual of a data point from the regression line. (c) Relative CpG methylation of ecDNA compared to the chromosomal locus and nucleosome positioning by MNase-seq, zooming into indicated gene promoters. Regions shown correspond to differentially methylated regions in Figure 3d,e. Mean methylation frequencies and MNase-seq coverage were calculated in 100-bp windows sliding every $10 \mathrm{bp}$. Relative frequencies were quantified from standardized residuals for a linear regression model for mean frequencies on ecDNA vs chromosomal DNA (Methods). 

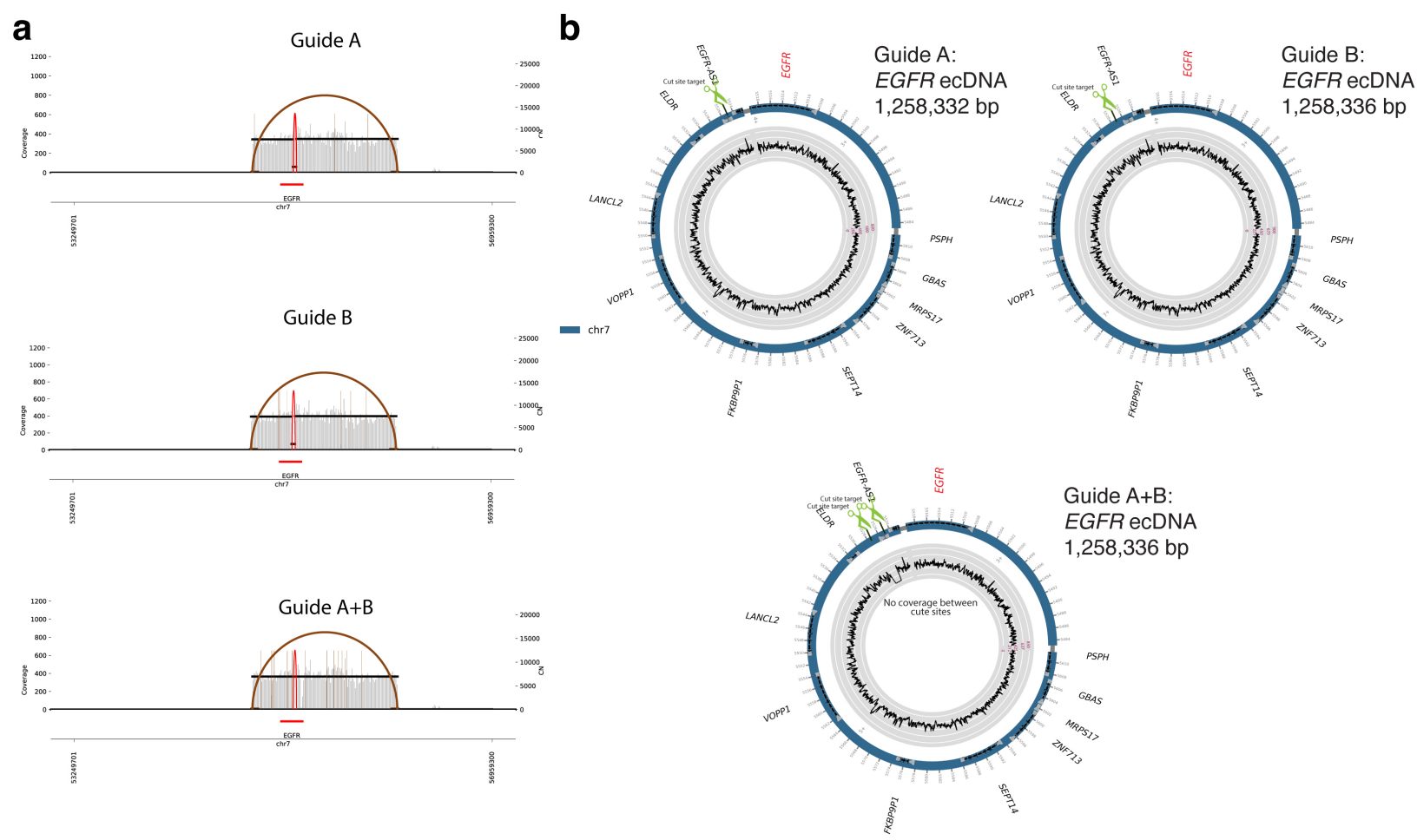

824 Extended Data Figure 3. Reconstruction of a $1.258 \mathrm{Mb}$ ecDNA from GBM39 825 neurospheres. (a) AmpliconArchitect breakpoint graphs for CRISPR-CATCH-purified ecDNAs using guides $A$ and/or $B$ as in Figure 1 (guide sequences in Supplementary Table 1). (b) Reconstructed ecDNA circles from CRISPR-CATCH data using independent sgRNAs showing equivalent ecDNA structures (outer rings; thin grey bands mark connections between sequence segments). Sequencing coverage is shown along the reconstructed circle (inner rings). Green scissors mark sgRNA target sites. Coordinate tick marks are printed in 10-kb units. AmpliconArchitect segment IDs and orientations are annotated. 
Hung et al., (CHANG), p32

a

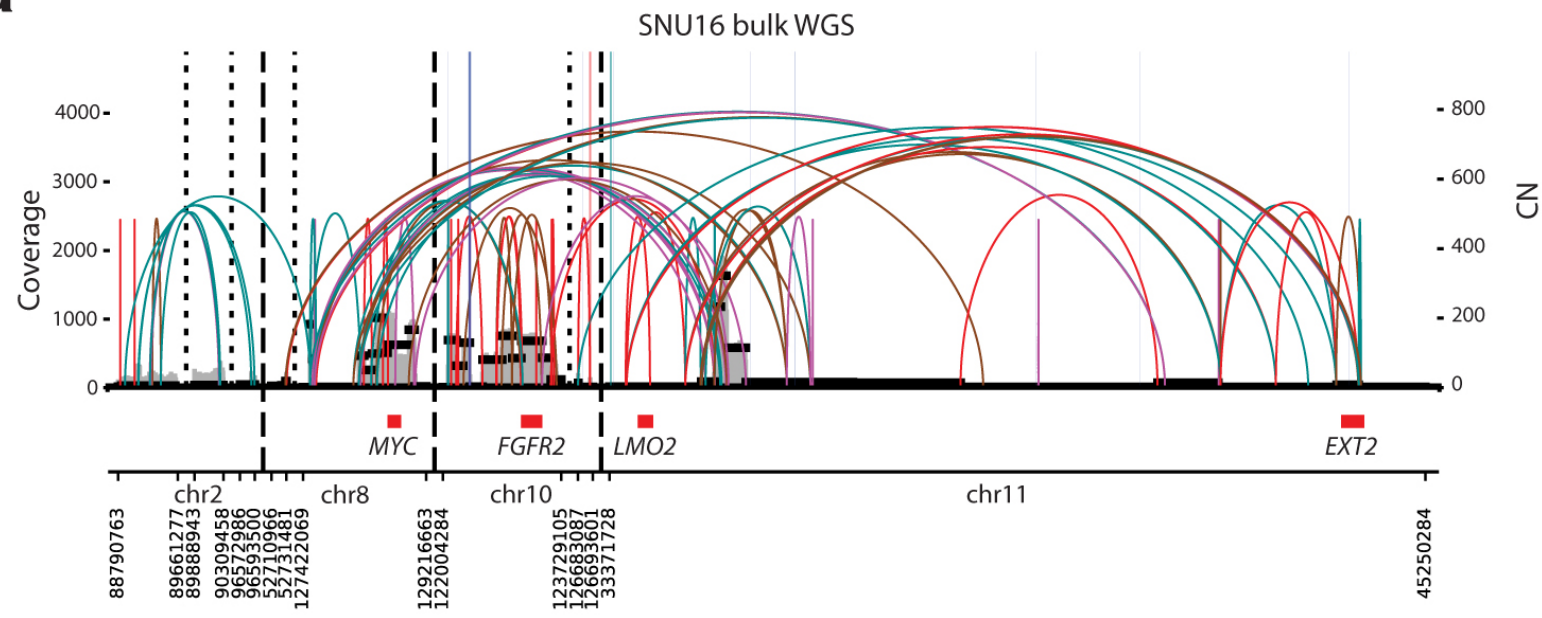

b

834

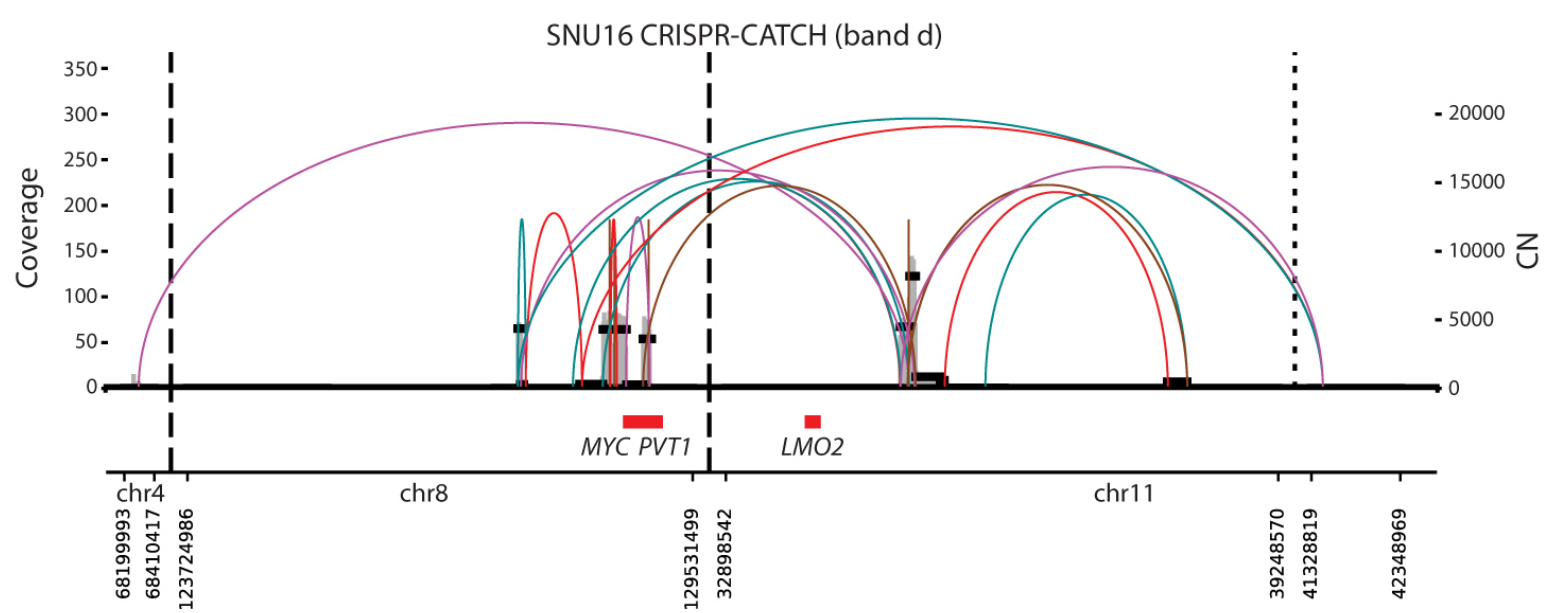

Extended Data Figure 4. CRISPR-CATCH enables disambiguation of heterogeneous sequence rearrangements on individual ecDNA species. (a) AmpliconArchitect breakpoint graph from bulk WGS of stomach cancer SNU16 cells showing significantly amplified sequences from chromosomes 8, 10, and 11. (b) An example of an AmpliconArchitect breakpoint graph for a CRISPR-CATCH-separated ecDNA species (band "d") from SNU16 cells showing greatly simplified breakpoints connecting only sequences from chromosomes 8 and 11. Gray vertical lines represent genomic coverage from WGS data and black horizontal lines indicate the estimated copy number of the region. Colored arcs represent breakpoint junctions, and the orientation of those junctions is specified by the color. Red and brown arcs preserve the orientation of the genome, with red reflecting breakpoints supported by reads in the proper orientation and brown reflecting breakpoints supported by reads in the everted orientation. Teal and magenta arcs indicate breakpoints leading to a change in genome orientation before and after the breakpoint where teal breakpoints are supported by both paired-end reads mapping to the forward strand and magenta breakpoints are supported by both pairedend reads mapping to the reverse strand. 
SNU16 CRISPR-CATCH bands:

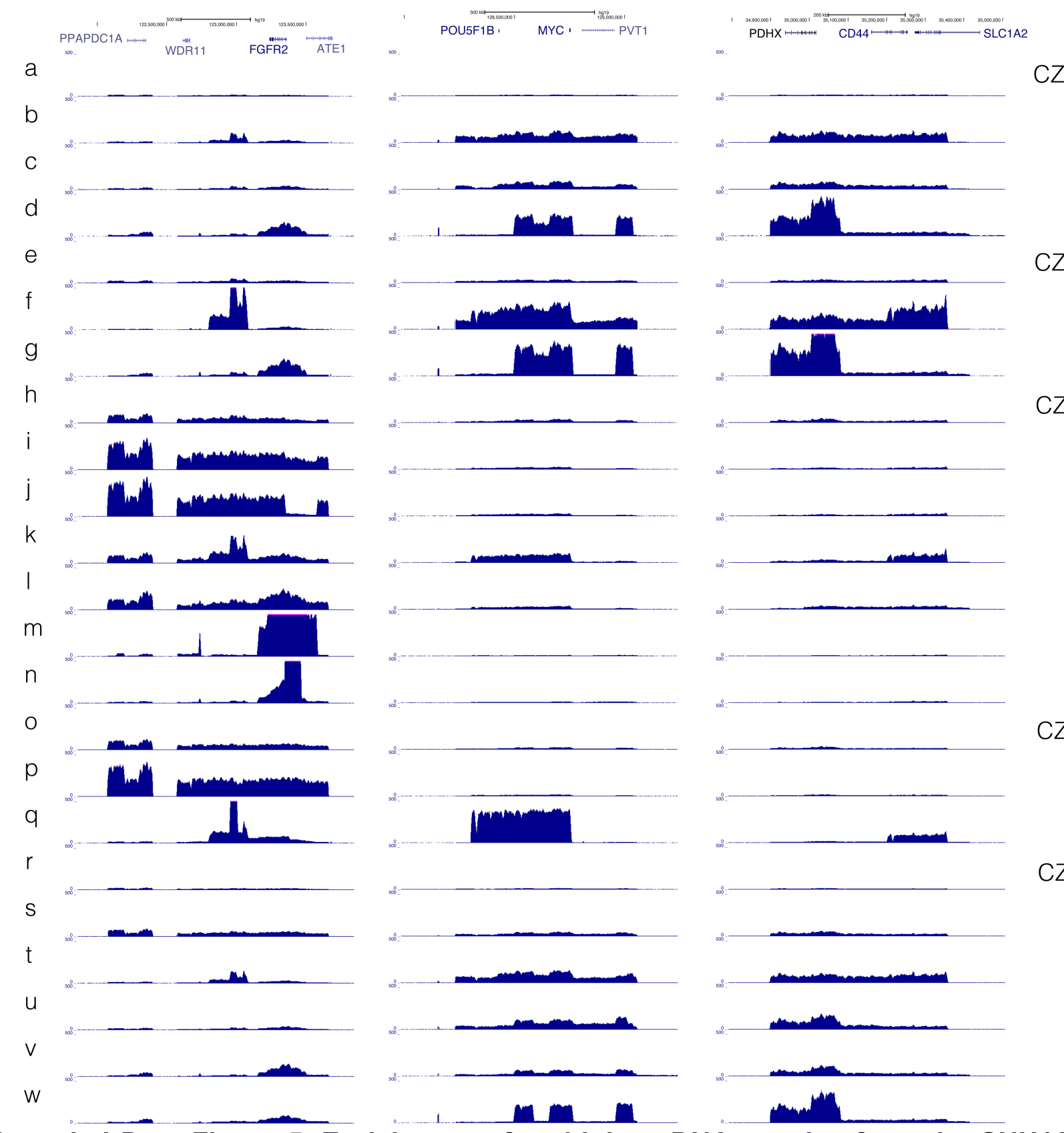
stomach cancer cell line. Short-read sequencing coverage tracks of multiple ecDNA species from SNU16 cells after CRISPR-CATCH purification at the FGFR2, MYC and CD44 loci. Bands a-w correspond to extracted bands shown in Figure 4b. Bands corresponding to unresolved DNA content in the compression zone are labeled CZ. 